\title{
CONVEX INTEGRATION ARISING IN THE MODELLING OF SHAPE-MEMORY ALLOYS: SOME REMARKS ON RIGIDITY, FLEXIBILITY AND SOME NUMERICAL IMPLEMENTATIONS
}

\author{
ANGKANA RÜLAND, JAMIE M TAYLOR, AND CHRISTIAN ZILLINGER
}

\begin{abstract}
We study convex integration solutions in the context of the modelling of shape-memory alloys. The purpose of the article is two-fold, treating both rigidity and flexibility properties: Firstly, we relate the maximal regularity of convex integration solutions to the presence of lower bounds in variational models with surface energy. Hence, variational models with surface energy could be viewed as a selection mechanism allowing for or excluding convex integration solutions. Secondly, we present the first numerical implementations of convex integration schemes for the model problem of the geometrically linearised two-dimensional hexagonal-to-rhombic phase transformation. We discuss and compare the two algorithms from [RZZ16] and [RZZ17] and give a numerical estimate of the regularity attained.
\end{abstract}

\section{INTRODUCTION}

Shape-memory alloys are materials undergoing a first order, diffusionless solidsolid phase transformation in which symmetry is lost. Here typically the high temperature phase, the austenite, is highly symmetric (e.g. having atomistically a highly symmetric unit cell structure), while the low temperature phase, the martensite, loses some of this symmetry. This loss of symmetry amounts to the presence of multiple, energetically equivalent variants of martensite. This renders these materials rather complex and gives rise to many complicated, interesting microstructures in these materials [Bha03].

1.1. The Ball-James variational model. Mathematically, various features of the microstructures in shape-memory alloys have been quite successfully modelled by (static) energy minimisation [BJ89, Kha13]:

$$
\min _{y(x)=M(x) \text { on } \partial \Omega} \int_{\Omega} W(\nabla y, \theta) d x .
$$

Here $\Omega \subset \mathbb{R}^{3}$ denotes the reference configuration (which is often chosen to be the material configuration in the austenite phase at fixed temperature), $y: \Omega \rightarrow$ $\mathbb{R}^{3}$ denotes the deformation of the material which is subjected to the boundary conditions $y(x)=M(x)$ on $\partial \Omega$. The function $\theta: \Omega \rightarrow \mathbb{R}_{+}$represents temperature (in normalised units) and $W: \mathbb{R}_{+}^{3 \times 3} \rightarrow \mathbb{R}_{+}$the stored energy function. Physical requirements on $W$ are:

(i) frame indifference: $W(Q M, \theta)=W(M, \theta)$ for each $M \in \mathbb{R}_{+}^{3 \times 3}, Q \in S O(3)$ and $\theta \in \mathbb{R}_{+}$.

(ii) material symmetry: $W(M H, \theta)=W(M, \theta)$ for each $M \in \mathbb{R}_{+}^{3 \times 3}, \theta \in \mathbb{R}_{+}$ and $H \in \mathcal{P}$, where $\mathcal{P} \subset S O(3)$ denotes the point group of the material, which encodes the symmetries of the material.

The symmetry of the phase transformation in conjunction with these two conditions then allows one to describe the energy wells of $W$ for a martensitic phase 
transformation

(2)

$W(M, \theta)=0 \Leftrightarrow M \in K(\theta)=\left\{\begin{array}{l}\alpha(\theta) S O(3) \text { if } \theta>\theta_{c}, \\ \bigcup_{j=1}^{m} S O(3) U_{j}(\theta) \text { if } \theta \leq \theta_{c},\end{array} \quad\right.$ with $U_{j}(\theta)^{t}=U_{j}(\theta)$.

Here $\theta_{c} \in \mathbb{R}_{+}$denotes the transformation temperature between the austenite and the martensite, $\alpha(\theta) S O(3)$ with $\alpha: \mathbb{R}_{+} \rightarrow \mathbb{R}_{+}$models the austenite phase at temperature $\theta$ with its thermal expansion $\alpha(\theta)$, and the matrices $S O(3) U_{j}(\theta)$ model the (energetically) equivalent variants of martensite at temperature $\theta \in \mathbb{R}_{+}[$Bal04]. The two conditions (i), (ii) render the minimisation problem (1) highly non-(quasi)convex and hard to analyse mathematically (even at a fixed temperature, which is the usual mathematical set-up) [Bal76, Bal02].

1.2. The $m$-well problem. As a consequence of the difficulty of dealing with the full problem (1), if one is interested in studying low energy microstructures, it is often useful to investigate exactly stress-free deformations and microstructures. These are the solutions to the differential inclusion

$$
\nabla y \in K(\theta) \text { a.e. in } \Omega, y(x)=M(x) \text { on } \partial \Omega,
$$

where $K(\theta)$ is the set from (2). If $\theta<\theta_{c},(3)$ is also called an $m$-well problem (due to the presence of $m$ variants of martensite). A natural question here is the existence of solutions to (3), respectively, the existence of energy zero solutions to (1). While highly oscillatory minimising sequences with limiting energy zero had been shown to exist for a large class of boundary data (related to the quasiconvex hull of $K(\theta)$; in general these sequences however only have measure valued limits), [BJ89, Mül99], the existence of exact energy zero solutions was only resolved later by relying on the technique of convex integration [MŠ99, MŠ98, DM12, Kir03, DKMŠ00, Gro73]. These quite surprising solutions however are rather "wild" and oscillatory in that they use a cascade of different scales (similar to convex integration solutions in fluid mechanics and geometry, see [SJ12] and the references therein). This "wildness" can be quantified in terms of the regularity of the solutions to (3). In particular, it has been shown in different models [DM95b, DM95a, Kir98, DKMŠ00, Kir98, Rül16a] that convex integration solutions can only exist at rough regularities, giving rise to a dichotomy. For instance for the two-well problem [DM95b, DM95a] (where there are exactly two rank-one connections between the wells) the following is known:

(a) If a solution is $B V$ regular, i.e. if $\nabla y \in B V$, then $\nabla y$ is a simple laminate, i.e. it is (up to boundary effects) "one-dimensional" with a directional dependence which is determined by the structure of the set $K(\theta)$ from (2).

(b) For any fixed $\theta<\theta_{c}$ and $M \in \operatorname{int}\left(K(\theta)^{l c}\right)$, where $K(\theta)^{l c}$ denotes the lamination convex hull of $K(\theta)$, there exist solutions to (3). By default these solutions are $L^{\infty}$ regular, i.e. $\nabla y \in L^{\infty}$.

Thus, a natural question is whether there is a threshold between these two regimes in terms of a critical regularity threshold (similar to the case in fluid mechanics and geometry [SJ12, Ise16, BDLSJV17, CDLS12]). In contrast to the situation in fluid mechanics, the problem (3) does not have a natural scaling, which would help to indicate a critical threshold. In previous work together with B. Zwicknagl [RZZ16, RZZ17], we have hence studied the possibility of deriving higher regularity for the solutions from (b) for simplified "geometrically linearised" models. We emphasise that although these models are "geometrically linearised", they are still highly nonlinear since the main material nonlinearity, which is manifested in the existence of multiple wells, remains present. It is one of the purposes of this article to show that scaling laws yield upper bounds on the possible regularity of these 
solutions (and could be viewed as "viscosity approximations" of the limiting model). Moreover, in the hope of eventually comparing our mathematical findings with experimental results, we present numerical simulations and estimates of convex integration solutions for the simplest possible model setting. We explain this in more detail in the following.

1.3. Surface energy regularisation and scaling laws. While various important qualitative properties, e.g. the emergence of preferred twinning directions as well as the presence of clear volume fractions of certain variants of martensite, have been successfully explained by (1) and (3), more quantitative properties such as the emergence of length scales are not captured by this. In the literature (see for instance [KM94, CO12, CO09, Rül16b, Con00, CC15, KKO13]) this has been remedied by adding a higher order surface energy contribution to (1), which is weighted with a small (material dependent) prefactor $\epsilon \in \mathbb{R}_{+}$:

$$
\min _{y(x)=M(x) \text { on } \partial \Omega}\left(\int_{\Omega} W(\nabla y, \theta) d x+\epsilon^{2} \int_{\Omega}\left|\nabla^{2} y\right|^{2} d x\right) .
$$

From a mathematical point of view the presence of the higher order term in (4) regularises the problem and restores compactness to it. In general, the exact physical form of the higher order term in (4) is not known and various types of different regularisations have been used in the literature, including the diffuse, $L^{2}$ variant stated in (4), but also the sharp, $B V$ variant (see [BMC09] for an overview on different models for this). In a sense (4) can be viewed as a selection criterion for certain microstructures that arise in (1) (and (3)); the additional surface term in (4) distinguishing between wild, rough and highly oscillatory (approximate) solutions in (1) and (approximate) solutions to (1), which are more regular (and hence use less surface energy). The properties of minimisers are often analysed by studying scaling limits of the functional (4), see for instance [Con00, KM94, CO12, CO09, Rül16b, Con00, CC15, KKO13]. While not predicting the exact form of the selected minimisers in (1), scaling does yield important information on these. Recently, a more precise limiting analysis has been carried out in [Sim17]. In spite of the various impressive advances in this direction, many points remain open in this context.

1.4. The main results. The purpose of this note is two-fold and addresses both rigidity and flexibility properties of the underlying differential inclusions.

1.4.1. Rigidity through scaling laws. Firstly, we show that scaling laws complement the analysis of convex integration solutions in a precise sense, in that the presence of a scaling law (or rather a lower bound on the energy (4)) yields an upper bound on the maximal regularity of convex integration solutions (which might be well-known to experts in the field, but which we could not find in the literature). Here we exploit that, while (3) itself does not have a prescribed scale predicting a possibly critical regularity, the model (4) has. In order to illustrate this interaction as clearly as possible, we here focus on the simplest possible type of stored energy function at fixed temperature $W(\nabla y, \theta)=\operatorname{dist}^{2}(\nabla y, K)$, which is motivated, though not justified, by a Taylor approximation around the wells.

Theorem 1. Let $\Omega \subset \mathbb{R}^{n}$ be an open bounded $C^{1,1}$ domain. Let

$$
\mathcal{E}_{\epsilon}(u):=\int_{\Omega} \operatorname{dist}^{2}(\nabla u, K) d x+\epsilon^{2} \int_{\Omega}\left|\nabla^{2} u\right|^{2} d x,
$$


where $K=K(\theta)$ for some fixed $\theta \in \mathbb{R}_{+}$is as in (2), and define

$$
E_{\epsilon, M}=\min _{\nabla u=M \text { a.e. in } \mathbb{R}^{n} \backslash \bar{\Omega}} \mathcal{E}_{\epsilon}(u) .
$$

Assume that there exists a solution $u$ to the differential inclusion

$$
\begin{aligned}
& \nabla u \in K \text { a.e. in } \Omega, \\
& \nabla u=M \text { a.e. in } \mathbb{R}^{n} \backslash \bar{\Omega},
\end{aligned}
$$

such that $v(x):=u(x)-M x-b \in H^{1+s}\left(\mathbb{R}^{n}\right)$ for some $b \in \mathbb{R}^{n}, s \in \mathbb{R}$ with $\operatorname{supp}(v) \subset \bar{\Omega}$. Then there exists a constant $C=C(M, \Omega, n, s)>1$ such that

$$
E_{\epsilon, M} \leq C \epsilon^{2 s} .
$$

In our context of convex integration solutions, we mainly seek to apply this result in its negation: If there is a lower scaling bound of the form $E_{\epsilon, M} \geq C \epsilon^{2 s}$ for some constant $C>0$ which is independent of $\epsilon$, then any solution to (7) with $\nabla u \in L^{\infty}\left(\mathbb{R}^{n}\right)$ has at best a Sobolev regularity of the form $\nabla u-M \in H^{s}\left(\mathbb{R}^{n}\right)$. Hence, a lower scaling bound yields an upper regularity threshold for the presence of convex integration solutions.

Remark 1.1. We remark that while working with displacement boundary conditions both in the original formulations of the energy (1) and the inclusion problem (3), we have formulated (7) with constant gradient in the exterior. As we consider the setting of affine boundary conditions, we can always switch between this and the displacement data problem.

Results which are similar to Theorem 1 can be obtained by using different forms of surface energies (see Propositions 2.2 and 2.4 in Section 2) and the corresponding Sobolev and Besov spaces. This is of physical relevance, as in general, the precise form of the surface energy contribution is not known and thus various different models have been proposed and analysed in the literature. These for instance include diffuse, Ginzburg-Landau and sharp interface energies, see [BMC09] for an overview on different models involving surface energies. In investigating different types of surface energy models, we show that the argument of Theorem 1 is very robust and does not depend on the specific model at hand (and can also be extended to the setting of geometrically linear elasticity, c.f. Remark 2.1).

The relevance of a result as in Theorem 1 stems from the interpretation of the surface energy regularised model: Since surface energies introduce a length scale in the model, it penalises too high oscillations. Hence, a surface energy regularised model can be viewed as a "viscosity" or "entropy" type regularisation of the original highly degenerate model. Thus, one might hope that such a regularised model provides a selection mechanism for the "physically relevant" solutions, which is reminiscent of regularisations of scalar conservation laws, for instance. As a consequence, it is of importance to study its relation to convex integration solutions. In particular, the result of Theorem 1 can be viewed as prescribing bounds on the maximal possible "fractality" (and fractal dimension) of possible solutions. This in turn leads to many interesting open questions on the study of "high regularity convex integration solutions". Here one of the most significant problems might be the optimal regularity which can be achieved in these constructions.

In order to illustrate the applicability of the deduced relation between scaling and regularity of convex integration solutions, we complement Theorem 1 by a lower, model-independent scaling bound. ${ }^{1}$

\footnotetext{
${ }^{1}$ We remark that we do not claim originality at this point, the addition of the lower bound stated in Theorem 2 and a sketch of its proof was suggested to us by one of the referees whom we would like to thank for this.
} 
Theorem 2. Let $\Omega \subset \mathbb{R}^{n}$ and $\mathcal{E}_{\epsilon}(u)$ be as in Theorem 1. Then for all $u: \mathbb{R}^{n} \rightarrow \mathbb{R}^{n}$ with $\nabla u=M$ in $\mathbb{R}^{n} \backslash \bar{\Omega}$ there exists a constant $C=C(\Omega, n, K)>0$ such that

$$
\mathcal{E}_{\epsilon}(u) \geq C \operatorname{dist}^{2}(M, K) \epsilon .
$$

Combining Theorems 1 and 2 immediately entails that on an $H^{s}$ scale, convex integration solutions cannot have gradients which are more than $H^{\frac{1}{2}}(\Omega)$ regular. This is in accordance with the regularity required for the presence of trace estimates (on an $L^{2}$ scale): As many interesting boundary data $M \in \mathbb{R}^{n \times n}$ are not contained in $K$, one expects that there is an infinite refinement in the values of $\nabla u$ towards the boundary of $\Omega$ (in order to accommodate the boundary conditions in a weak sense). In particular, one expects (with very few exceptions, see [RZZ16, Section 7] and [CKZ17]) that $\nabla u$ does not have a (two-sided and coinciding) trace at the boundary (but might rather be in function spaces allowing for jumps or not even proper traces at all). We emphasise that, due to its model independence, the lower bound of Theorem 2 is applicable to a large class of problems. In terms of scaling it is (essentially) optimal in the case $K=O(2)$, which follows from the constructions of [DMP08b, DMP08a, DMP10]. However, for most of the situations that we are interested in and which are motivated by differential inclusions in martensitic phase transformations, it seems unlikely that the bound from Theorem 2 is sharp. Indeed, the following estimates are known in interesting (but non-exhaustive) model cases:

- In the Kohn-Müller model [KM94] which is a scalar model problem for shape-memory alloys a scaling of $\epsilon^{\frac{2}{3}}$ is proved for an austenite-martensite interface in two dimensions. However, in this problem no convex integration solutions exist.

- In [CC15] a two-dimensional two-well problem with austenite boundary conditions (i.e. identity boundary conditions) is considered. In the case of two rank-one connections between the two wells (and non-degenerate domains) the authors prove a scaling of $\epsilon^{\frac{2}{3}}$, and a scaling of $\epsilon^{\frac{4}{5}}$ in the case of one rank-one connection. This would correspond to an upper regularity bound for convex integration solutions of the scale $H^{\frac{1}{3}}$ and $H^{\frac{2}{5}}$, respectively (however, it is possible to show that there are no convex integration solutions for identity boundary data [Ped97, Chapter 3]).

- In a geometrically linear model for the cubic-to-tetragonal phase transformation, the results of [CO12, CO09] prove $\epsilon^{\frac{2}{3}}$ scaling. However, also in this model there are no convex integration solutions [Sim17].

While none of the described models can be applied in the setting with convex integration solutions, the described lower bounds strongly suggest that also in the convex integration setting the critical function spaces on an $H^{s}$ scale for relevant phase transformations are below the $H^{\frac{1}{2}}$ threshold from Theorem 2 (and most likely also below the $H^{\frac{1}{3}}$ threshold from the above examples, as one expects the microstructure to be more complex in the presence of convex integration solutions than in the case without convex integration solutions).

1.4.2. Numerical analysis of flexible solutions. Secondly, in addition to providing the described upper bound on the maximal regularity of convex integration solutions in terms of the behaviour of scaling laws, we present numerical implementations of the convex integration schemes discussed in [RZZ16] and [RZZ17] in the case of the model setting of the geometrically linearised hexagonal-to-rhombic phase transformation (see Section 3.1 for a more detailed description of this phase transformation). Here we pursue two main objectives: On the one hand, by for the first time presenting explicit pictures of convex integration solutions in elasticity, we hope to attract interest in convex integration solutions from more experimentally oriented 
researchers. We hope that the explicit images eventually provide an opportunity to compare our theoretical findings with experiments (we refer to [KK91, CPL14] for experimental results on the considered phase transformation and to the work of Inamura [Ina] for first experimental results pointing into the direction that convex integration solutions might indeed be of physical significance). On the other hand, we aim at illustrating the fractal character of the solutions computed by our convex integration schemes, indicating that at least with the present convex integration algorithms one would expect regularity thresholds which are relatively low. We complement this rather qualitative study of the numerics of convex integration solutions by a more quantitative analysis by presenting the distribution of length scales in our numerical constructions. This is intimately tied to regularity estimates (through the growth of the surface energies).

Although treating the "flexible" side of the convex integration problem, this second part of our work is closely connected to the "rigidity" result on the critical regularity from Theorem 1. Indeed, in both results, we seek to understand the "fractality" of possible solutions: The articles [RZZ16, RZZ17] provided lower bounds on the fractal dimension and regularity of convex integration solutions. However, as explained in these articles, the main purpose there was to establish some qualitative degree of higher regularity of convex integration solutions in elasticity, which had not been known to be possible previously. While we sought to optimise the qualitative dependences of the regularity exponents in [RZZ16, RZZ17] (e.g. in trying to understand how the regularity depends on the distance of the affine boundary data to the boundary of the convex hull), we did not try to optimise the quantitative properties of these constructions (e.g. the factors in our interpolation arguments). As a result, the rigorously derived exponents in [RZZ16, RZZ17] only yield a lower bound on the real regularity of convex integration solutions. We expect that they underestimate the "real" regularities by several orders of magnitude (this is probably particularly striking in the setting of [RZZ16], where we use extremely carefully crafted covering arguments to estimate the resulting $B V$ norms and in which we give up a lot on the "helpful" volume fraction $v$ on which the deformation becomes "good" in each iteration step). Moreover, careful numerical implementations also seem to confirm our expectation (based on theoretical estimates) that in the setting of the convex integration scheme from [RZZ16] the position of the boundary data in matrix space has a non-negligible influence on the regularity of the resulting convex integration solutions (see Figure 12). Hence, we believe that it is important to provide an implementation of the corresponding schemes in order to achieve a better understanding of the possible real fractal dimensions.

All in all a common objective in both of our results is to improve the understanding of the scales and the "fractal nature" which underlie convex integration solutions in the mathematical modelling of shape-memory alloys. While not answering the very ambitious question of whether these solutions are of physical relevance, our discussion does provide a partial answer to the more modest mathematical problem of deducing a more detailed understanding of the scales which are involved in convex integration solutions. We believe that the better understanding of the mathematics of convex integration solutions could also help in deriving an improved understanding of their possible physical significance.

1.5. Outline of the article. The remainder of the article is organised as follows: First, in Section 2, we address the relation between scaling laws and rigidity results. In particular, we present the proof of Theorem 1 (and discuss variants of it). Next, in Section 3, we briefly recall the convex integration algorithms from [RZZ16] and [RZZ17] for the model case of the hexagonal-to-rhombic phase transformation and present numerical implementations of these. This includes illustrations of the 
resulting convex integration solutions (Section 3.3) of which we hope that they contribute to eventually bridge the gap between experiment and theory and more quantitative investigations of the length scale distributions of convex integration solutions (Section 3.4).

Acknowledgements. During the research leading to these results Jamie M. Taylor held positions at the University of Oxford, at Kent State University and BCAM. His research received funding from the European Research Council under the European Union's Seventh Framework Programme (FP7/2007-2013)/ERC grant agreement no 291053. Furthermore, he has been partially supported by the Basque Government through the BERC 2018-2021 program; and by Spanish Ministry of Economy and Competitiveness MINECO through BCAM Severo Ochoa excellence accreditation SEV-2017-0718 and through project MTM2017-82184-R funded by (AEI/FEDER, UE) and acronym "DESFLU". Christian Zillinger acknowledges the support of an AMS-Simons Travel Grant.

\section{Rigidity AND SCALing LAWs}

In this section we present the derivation of the bound on the maximal possible regularity of convex integration solutions. Here we do not yet specify the set-up to that of a particular phase transformation but remain in the general framework, which was layed out in Section 1.

We explain the close relation between scaling laws and the possible regularity of convex integration solutions (which is reminiscent of the mathematical analysis of turbulence in fluids for which we refer to [Fri95, SJ12]): Assume that for a bounded Lipschitz domain $\Omega \subset \mathbb{R}^{n}$ a function $u \in W^{1, \infty}(\Omega)$, which in addition satisfies $\nabla u \in H_{l o c}^{\gamma}\left(\mathbb{R}^{n}\right)$, is a solution to the differential inclusion

$$
\begin{aligned}
& \nabla u \in K \text { a.e. in } \Omega, \\
& \nabla u=M \text { a.e. in } \mathbb{R}^{n} \backslash \bar{\Omega},
\end{aligned}
$$

where $K:=K(\theta)$ denotes the wells from (2) for some fixed temperature $\theta>0$ (this corresponds to the case of affine boundary data in (3), which is already an interesting case). Then, any sequence $\nabla u_{k}$ in $H_{l o c}^{\gamma}\left(\mathbb{R}^{n}\right)$ with $\nabla u_{k} \rightarrow \nabla u$ in $L_{l o c}^{2}\left(\mathbb{R}^{n}\right)$ and with $\nabla u_{k}=M$ a.e. in $\mathbb{R}^{n} \backslash \bar{\Omega}$ is an admissible competitor in the minimisation problem for $E_{\epsilon, M}$, where $E_{\epsilon, M}$ is the energy from (6). We use this observation to prove that a lower bound in the scaling of the minimal energy $E_{\epsilon, M}$ then yields a bound on the maximal regularity of $\nabla u$.

Let us explain the heuristics of the derivation of the upper scaling bounds assuming $H^{s}$ regularity of $\nabla u \in K$ in slightly more detail. To this end, we introduce a regularised version $u_{\delta}$ of $u$, the details of which will be described later, which is still admissible in the minimisation problem for the minimal energy $E_{\epsilon, M}$. Then, as $\nabla u \in K$ and by definition of the distance, we have

$$
\mathcal{E}_{\epsilon}\left(u_{\delta}\right) \leq 2\left(\left\|\nabla u_{\delta}-\nabla u\right\|_{L^{2}(\Omega)}^{2}+\epsilon^{2}\left\|\nabla^{2} u_{\delta}\right\|_{L^{2}(\Omega)}^{2}\right) .
$$

Next we argue by interpolation: From scaling arguments, where $\delta$ is treated as a lengthscale and where we ignore boundary effects, we expect that for a constant $C>0$ which is independent of $\delta$

$$
\begin{aligned}
\left\|\nabla u_{\delta}-\nabla u\right\|_{L^{2}\left(\mathbb{R}^{n}\right)} & \leq C \delta^{s}\left\||\nabla|^{s} \nabla u\right\|_{L^{2}\left(\mathbb{R}^{n}\right)}, \\
\left\|\nabla^{2} u_{\delta}\right\|_{L^{2}\left(\mathbb{R}^{n}\right)} & \leq C \delta^{s-1}\left\||\nabla|^{s} \nabla u\right\|_{L^{2}\left(\mathbb{R}^{n}\right)} .
\end{aligned}
$$

Inserting these bounds into (8) then yields

$$
\mathcal{E}_{\epsilon}\left(u_{\delta}\right) \leq C\left(\delta^{2 s}+\epsilon^{2} \delta^{2 s-2}\right)\left\||\nabla|^{s} \nabla u\right\|_{L^{2}\left(\mathbb{R}^{n}\right)}^{2} .
$$


Optimising the right hand side in $\delta$ by choosing $\delta \sim \epsilon$ results in the claimed scaling bound provided $\nabla u \in \dot{H}^{s}(\Omega)$. Similar arguments indicate "critical" scaling behaviour matching the assumed regularity of solutions to (7), if the energies in (6) are not diffuse, but for instance sharp interface models.

The above described intuition can be made rigorous and directly yields the proof of Theorem 1 (see Section 2.2).

2.1. Function spaces. Before proceeding with the discussion of the relation between regularity and scaling, for reference we first collect the function spaces, which we will be using in the sequel. Denoting the Fourier transform by $\mathcal{F} w(k)=$ $\int_{\mathbb{R}^{n}} e^{i k \cdot x} w(x) d x$, for $s \in \mathbb{R}$ we consider the $L^{2}$ based fractional Sobolev spaces

$$
H^{s}\left(\mathbb{R}^{n}\right):=\left\{u \in \mathcal{D}^{\prime}\left(\mathbb{R}^{n}\right):\|u\|_{H^{s}\left(\mathbb{R}^{n}\right)}:=\left\|\mathcal{F}^{-1}\left[\left(|\xi|^{2}+1\right)^{s / 2} \mathcal{F} u\right]\right\|_{L^{2}\left(\mathbb{R}^{n}\right)}<\infty\right\} .
$$

Moreover, we will also use Besov spaces. To this end we work with a LittlewoodPaley decomposition. Following [Tao06, Appendix A], we let $\varphi$ denote a nonnegative, radially symmetric bump function supported in $\left\{k \in \mathbb{R}^{d}:|k| \leq 2\right\}$, which is equal to one on $\left\{k \in \mathbb{R}^{d}:|k| \leq 1\right\}$. Let $N \in 2^{\mathbb{Z}}$, i.e. assume that there exists $j \in \mathbb{Z}$ such that $N=2^{j}$. We then define the Littlewood-Paley projectors $P_{<N}, P_{\geq N}, P_{N}$ to be

$$
\begin{aligned}
\mathcal{F}\left(P_{<N} f\right)(k) & :=\varphi(k / N) \mathcal{F} f(k), \\
\mathcal{F}\left(P_{\geq N} f\right)(k) & :=(1-\varphi(k / N)) \mathcal{F} f(k), \\
\mathcal{F}\left(P_{N} f\right)(k) & :=(\varphi(k / N)-\varphi(2 k / N)) \mathcal{F} f(k) .
\end{aligned}
$$

With this in hand, we recall that for $s \in \mathbb{R}$ and $p \in[1, \infty)$ the space of Besov functions $f \in B_{p, p}^{s}\left(\mathbb{R}^{n}\right)$ is defined as

$$
B_{p, p}^{s}\left(\mathbb{R}^{n}\right)=\left\{f \in \mathcal{D}^{\prime}\left(\mathbb{R}^{n}\right):\|f\|_{B_{p, p}^{s}\left(\mathbb{R}^{n}\right)}<\infty\right\},
$$

where

$$
\|f\|_{B_{p, p}^{s}\left(\mathbb{R}^{n}\right)}:=\left(\sum_{N \in 2^{\mathbb{Z}}} N^{s p}\left\|P_{N} f\right\|_{L^{p}\left(\mathbb{R}^{n}\right)}^{p}\right)^{1 / p},
$$

see for instance [Tri06] or [BCD11]. For later use, we recall that $B_{1,1}^{0}\left(\mathbb{R}^{n}\right) \subset L^{1}\left(\mathbb{R}^{n}\right)$ (see Theorem 2.41 in $[\mathrm{BCD} 11]$ ). For $s>0, s \notin \mathbb{N}$ the space $B_{p, p}^{s}\left(\mathbb{R}^{n}\right)$ coincides with the fractional Sobolev space $W^{s, p}\left(\mathbb{R}^{n}\right)$, which is defined by the norm

$$
\|u\|_{W^{s, p}\left(\mathbb{R}^{n}\right)}^{p}=\|u\|_{L^{p}\left(\mathbb{R}^{n}\right)}^{p}+\left\|\nabla^{k} u\right\|_{L^{p}\left(\mathbb{R}^{n}\right)}^{p}+\int_{\mathbb{R}^{n}} \int_{\mathbb{R}^{n}} \frac{\left|\nabla^{k} u(x)-\nabla^{k} u(y)\right|^{p}}{|x-y|^{n+\sigma p}} d y d x
$$

where $s=k+\sigma, k \in \mathbb{N}, \sigma \in(0,1)$ (see [BM01] for a comparison of several fractional Sobolev type function spaces which are defined by equivalent, but slightly different norms).

We conclude our discussion on function spaces and Littlewood-Paley decompositions by noting that the low frequency projection in Littlewood-Paley theory is really only a (special type of) convolution:

$$
v_{<N}(x):=\left(P_{<N} v\right)(x)=\left(\mathcal{F}^{-1}\left(\varphi\left(\frac{\cdot}{N}\right)\right) * v\right)(x),
$$

where $\varphi$ is the bump function from (10) and $v \in C_{0}^{\infty}\left(\mathbb{R}^{n}\right)$. Moreover, by the scaling behaviour of the Fourier transform, we have

$$
\left(\mathcal{F}^{-1} \varphi\left(\frac{\cdot}{N}\right)\right)(x)=N^{n}\left(\mathcal{F}^{-1} \varphi\right)(N x) .
$$

Thus, regularisation by frequency cut-off is a special type of regularisation by convolution. Instead of convolving with a function which is compactly supported in 
real space, we convolve with a function which is compactly supported in Fourier space. However, also in real space this is a Schwartz function, so in particular it is rapidly decaying at infinity, thus mainly giving weight to localized information.

As a consequence of these remarks, we infer that for any $p \in[1, \infty]$ we have

$$
\left\|v_{<N}\right\|_{L^{p}\left(\mathbb{R}^{n}\right)}+\left\|v_{\geq N}\right\|_{L^{p}\left(\mathbb{R}^{n}\right)} \leq C\|v\|_{L^{p}\left(\mathbb{R}^{n}\right)}
$$

where $C>0$ is a constant that depends on $p, n, \varphi$, with $\varphi$ being the bump function from the definition of the Littlewood-Paley projection (10). The bounds in (13) will be frequently used in the sequel.

These bounds can be obtained by the convolution structure of the LittlewoodPaley decomposition: Indeed, using the convolution representation (11) of $v_{<N}$ in conjunction with (12), Young's convolution inequality implies the estimate

$$
\left\|v_{<N}\right\|_{L^{p}\left(\mathbb{R}^{n}\right)} \leq C\left\|N^{n}\left(\mathcal{F}^{-1} \varphi\right)(N \cdot)\right\|_{L^{1}\left(\mathbb{R}^{n}\right)}\|v\|_{L^{p}\left(\mathbb{R}^{n}\right)} \leq C_{\varphi}\|v\|_{L^{p}\left(\mathbb{R}^{n}\right)}
$$

for any $p \in[1, \infty]$. Hence, by the triangle inequality,

$$
\left\|v_{\geq N}\right\|_{L^{p}\left(\mathbb{R}^{n}\right)} \leq\|v\|_{L^{p}\left(\mathbb{R}^{n}\right)}+\left\|v_{<N}\right\|_{L^{p}\left(\mathbb{R}^{n}\right)} .
$$

Therefore, by (14) this then also entails that

$$
\left\|v_{\geq N}\right\|_{L^{p}\left(\mathbb{R}^{n}\right)} \leq C_{\varphi}\|v\|_{L^{p}\left(\mathbb{R}^{n}\right)} \text { for any } p \in[1, \infty],
$$

which concludes the argument for (13).

2.2. Proof of Theorem 1. We begin the discussion of the relation between scaling and regularity by presenting the proof of Theorem 1 . In this context of $L^{2}$ based energies, we present two proofs for Theorem 1, one based on LittlewoodPaley decomposition and frequency truncation and one based on convolution. The first proof is very convenient in extending our arguments to different (Besov type) function spaces, while the second argument might be more in line with the literature on scaling laws. We emphasise that here and in the following proofs the constants in the estimates will be generic and may change from line to line.

Proof of Theorem 1. We argue by regularisation (through a frequency cut-off in the corresponding Littlewood-Paley decomposition) and a spacial cut-off (in order to ensure the validity of the boundary conditions).

Let $u$ be a solution to (7). As a preliminary step, to simplify the set-up, we shift the problem (7) by introducing $v(x)=u(x)-M x-b$ for $b \in \mathbb{R}^{n}$, which solves

$$
\begin{aligned}
& \nabla v \in \tilde{K} \text { a.e. in } \Omega, \\
& \nabla v=0 \text { a.e. in } \mathbb{R}^{n} \backslash \bar{\Omega},
\end{aligned}
$$

where $\tilde{K}=K-M:=\{N-M: N \in K\}$. The constant $b \in \mathbb{R}^{n}$ is chosen such that $v \in H^{s+1}\left(\mathbb{R}^{n}\right)$ and $\operatorname{supp}(v) \subset \bar{\Omega}$. We seek to prove upper scaling bounds for the associated energy minimum. Rewritten in terms of the shifted functions $v$, the energy (6) turns into

$$
\tilde{E}_{\epsilon}=\min _{\nabla v=0 \text { a.e. in } \mathbb{R}^{n} \backslash \bar{\Omega}} \int_{\Omega} \operatorname{dist}^{2}(\nabla v, \tilde{K}) d x+\epsilon^{2} \int_{\Omega}\left|\nabla^{2} v\right|^{2} d x .
$$

For $P_{<N}, P_{\geq N}$ denoting the standard Littlewood-Paley projector (see the definition in $(10))$, in the sequel, we use the following notation:

$$
(\nabla u)_{<N}:=P_{<N}(\nabla u),(\nabla u)_{\geq N}:=P_{\geq N}(\nabla u) .
$$


Given the function $v$, we cut-off its high frequencies and correct the frequency cutoff of $v$ so that it satisfies the desired boundary conditions, i.e. we consider the function

$$
\tilde{v}_{N}(x):=\eta_{\delta}(x) v_{<N}(x),
$$

where $\eta_{\delta}$ is a smooth, positive cut-off function which is equal to one in $\Omega_{2 \delta}:=\{x \in$ $\Omega: \operatorname{dist}(x, \partial \Omega)>2 \delta\}$, which vanishes outside of $\Omega_{\delta}:=\{x \in \Omega: \operatorname{dist}(x, \partial \Omega)>\delta\}$ and which satisfies

$$
\left|\nabla \eta_{\delta}\right| \leq \frac{C}{\delta},\left|\nabla^{2} \eta_{\delta}\right| \leq \frac{C}{\delta^{2}}
$$

The value of the parameter $\delta$ will be determined in the sequel. Moreover, here and in the following arguments, the constant $C>0$ will be generic and might change from line to line, but will always be independent of $\delta, N, \epsilon$. We observe that for $v$ with $\nabla v \in H^{s}\left(\mathbb{R}^{n}\right)$ Bernstein estimates (see the Appendix in [Tao06], and Section 2.1.1. in [BCD11]) yield

$$
\begin{aligned}
\left\|\nabla^{2} v_{<N}\right\|_{L^{2}(\Omega)} & \leq\left\|\nabla^{2} v_{<N}\right\|_{L^{2}\left(\mathbb{R}^{n}\right)} \leq N^{1-s}\|\nabla v\|_{H^{s}\left(\mathbb{R}^{n}\right)}, \\
\left\|(\nabla v)_{\geq N}\right\|_{L^{2}(\Omega)} & \leq\left\|(\nabla v)_{\geq N}\right\|_{L^{2}\left(\mathbb{R}^{n}\right)} \leq N^{-s}\|\nabla v\|_{H^{s}\left(\mathbb{R}^{n}\right)} .
\end{aligned}
$$

We now argue in two steps and first estimate the size of $\left\|\operatorname{dist}\left(\nabla \tilde{v}_{N}, \tilde{K}\right)\right\|_{L^{2}(\Omega)}^{2}$. Afterwards we prove a bound for $\epsilon^{2}\left\|\nabla^{2} \tilde{v}_{N}\right\|_{L^{2}(\Omega)}^{2}$. Balancing these estimates, we will derive a bound on the possible scaling of $E_{\epsilon, M}$ in dependence of the regularity of $\nabla v$ (and hence for $\nabla u$ ).

Step 1: Estimate for $\left\|\operatorname{dist}\left(\nabla \tilde{v}_{N}, \tilde{K}\right)\right\|_{L^{2}(\Omega)}^{2}$. Using the triangle inequality, we estimate:

$$
\begin{aligned}
\left\|\operatorname{dist}\left(\nabla \tilde{v}_{N}, \tilde{K}\right)\right\|_{L^{2}(\Omega)}^{2} \leq & C\left(\left\|\operatorname{dist}\left(\eta_{\delta} \nabla v_{<N}, \tilde{K}\right)\right\|_{L^{2}(\Omega)}^{2}+\left\|v_{<N} \nabla \eta_{\delta}\right\|_{L^{2}(\Omega)}^{2}\right) \\
\leq & C\left(\|\operatorname{dist}(\nabla v, \tilde{K})\|_{L^{2}(\Omega)}^{2}+\left\|\left(1-\eta_{\delta}\right) \nabla v\right\|_{L^{2}(\Omega)}^{2}\right. \\
& \left.+\left\|\nabla v-\nabla v_{<N}\right\|_{L^{2}(\Omega)}^{2}+\left\|v_{<N} \nabla \eta_{\delta}\right\|_{L^{2}(\Omega)}^{2}\right) \\
\leq & C\left(\delta^{2 s}\|\nabla v\|_{H^{s}\left(\mathbb{R}^{n}\right)}^{2}+\left\|v_{<N} \nabla \eta_{\delta}\right\|_{L^{2}\left(\mathbb{R}^{n}\right)}^{2}\right. \\
& \left.+\left\|\nabla v-\nabla v_{<N}\right\|_{L^{2}\left(\mathbb{R}^{n}\right)}^{2}\right) .
\end{aligned}
$$

Here we used that the support condition for $\eta_{\delta}$ and Poincaré's inequality to bound

$$
\left\|\left(1-\eta_{\delta}\right) \nabla v\right\|_{L^{2}(\Omega)}^{2} \leq\|\nabla v\|_{L^{2}\left(\Omega \backslash \Omega_{2 \delta}\right)}^{2} \leq \delta^{2 s}\|\nabla v\|_{H^{s}\left(\mathbb{R}^{n}\right)}^{2} .
$$

We estimate the remaining two terms on the right hand side of (21) separately: First, we apply the Bernstein estimates (see (20)) to infer

$$
\left\|\nabla v-\nabla v_{<N}\right\|_{L^{2}\left(\mathbb{R}^{n}\right)}^{2}=\|\nabla v \geq N\|_{L^{2}\left(\mathbb{R}^{n}\right)}^{2} \leq C N^{-2 s}\|\nabla v\|_{H^{s}\left(\mathbb{R}^{n}\right)}^{2} .
$$

Next, we estimate

$$
\left\|v_{<N} \nabla \eta_{\delta}\right\|_{L^{2}\left(\mathbb{R}^{n}\right)}^{2} \leq C\left(\left\|v \nabla \eta_{\delta}\right\|_{L^{2}\left(\mathbb{R}^{n}\right)}^{2}+\left\|v_{\geq N} \nabla \eta_{\delta}\right\|_{L^{2}\left(\mathbb{R}^{n}\right)}^{2}\right) .
$$

In order to bound the second term on the right hand side of (23), we note that by the bounds for $\eta_{\delta}$ (see (19)) and Bernstein estimates

$$
\left\|v_{\geq N} \nabla \eta_{\delta}\right\|_{L^{2}\left(\mathbb{R}^{n}\right)}^{2} \leq C \delta^{-2}\left\|v_{\geq N}\right\|_{L^{2}\left(\mathbb{R}^{n}\right)}^{2} \leq C \delta^{-2} N^{-2-2 s}\|\nabla v\|_{H^{s}\left(\mathbb{R}^{n}\right)}^{2} .
$$

For the first term on the right hand side of (23), using that $\operatorname{supp}\left(\nabla \eta_{\delta}\right) \subset \Omega_{\delta} \backslash \Omega_{2 \delta}$, we obtain

$$
\left\|v \nabla \eta_{\delta}\right\|_{L^{2}\left(\mathbb{R}^{n}\right)}^{2} \leq C \delta^{-2}\|v\|_{L^{2}\left(\Omega_{\delta} \backslash \Omega_{2 \delta}\right)}^{2} \leq C\|\nabla v\|_{L^{2}\left(\Omega \backslash \Omega_{2 \delta}\right)}^{2} \leq C \delta^{2 s}\|\nabla v\|_{H^{s}\left(\mathbb{R}^{n}\right)}^{2} .
$$


Indeed, the last estimate in this chain of inequalities can be inferred by using the support assumption for $\nabla v$ and a fractional Poincaré inequality (see Lemma A.1 in Appendix A).

Combining the bounds from (22)-(25), we thus obtain as the estimate for the elastic energy

$$
\left\|\operatorname{dist}\left(\nabla \tilde{v}_{N}, \tilde{K}\right)\right\|_{L^{2}(\Omega)}^{2} \leq C\left(N^{-2 s}+\delta^{-2} N^{-2-2 s}+\delta^{2 s}\right)\|\nabla v\|_{H^{s}(\Omega)}^{2} .
$$

Step 2: Estimate for the surface energy and conclusion. Next we estimate the surface energy:

$$
\begin{aligned}
\left\|\nabla^{2} \tilde{v}_{N}\right\|_{L^{2}(\Omega)}^{2} & \leq C\left(\left\|\nabla^{2} v_{<N}\right\|_{L^{2}\left(\mathbb{R}^{n}\right)}^{2}+\left\|v_{<N} \nabla^{2} \eta_{\delta}\right\|_{L^{2}\left(\mathbb{R}^{n}\right)}^{2}+\left\|\nabla \eta_{\delta} \nabla v_{<N}\right\|_{L^{2}\left(\mathbb{R}^{n}\right)}^{2}\right) \\
& \leq C\left(N^{2-2 s}\|\nabla v\|_{H^{s}\left(\mathbb{R}^{n}\right)}^{2}+\left\|v_{<N} \nabla^{2} \eta_{\delta}\right\|_{L^{2}\left(\mathbb{R}^{n}\right)}^{2}+\left\|\nabla \eta_{\delta} \nabla v_{<N}\right\|_{L^{2}\left(\mathbb{R}^{n}\right)}^{2}\right) .
\end{aligned}
$$

We again estimate the two last terms separately: Using a fractional Poincaré inequality once more in combination with Bernstein estimates, we obtain for the third term in (27)

$$
\begin{aligned}
\left\|\nabla \eta_{\delta} \nabla v_{<N}\right\|_{L^{2}\left(\mathbb{R}^{n}\right)}^{2} & \leq C\left(\left\|\nabla \eta_{\delta} \nabla v\right\|_{L^{2}(\Omega)}^{2}+\left\|\nabla \eta_{\delta} \nabla v_{\geq N}\right\|_{L^{2}(\Omega)}^{2}\right) \\
& \leq C \delta^{-2} \delta^{2 s}\|\nabla v\|_{H^{s}\left(\mathbb{R}^{n}\right)}^{2}+C \delta^{-2} N^{-2 s}\|\nabla v\|_{H^{s}\left(\mathbb{R}^{n}\right)}^{2} .
\end{aligned}
$$

For the second term in (27), we similarly note by Poincaré and Bernstein

$$
\begin{aligned}
\left\|v_{<N} \nabla^{2} \eta_{\delta}\right\|_{L^{2}(\Omega)}^{2} & \leq C\left\|v \nabla^{2} \eta_{\delta}\right\|_{L^{2}\left(\mathbb{R}^{n}\right)}^{2}+C\left\|v_{\geq N} \nabla^{2} \eta_{\delta}\right\|_{L^{2}\left(\mathbb{R}^{n}\right)}^{2} \\
& \leq C\|\nabla v\|_{H^{s}\left(\mathbb{R}^{n}\right)}^{2}\left(\delta^{-2+2 s}+\delta^{-4} N^{-2-2 s}\right) .
\end{aligned}
$$

Combining (27)-(29) we therefore obtain

$$
\epsilon^{2}\left\|\nabla^{2} \tilde{v}_{N}\right\|_{L^{2}(\Omega)}^{2} \leq C \epsilon^{2}\left(N^{2-2 s}+\delta^{-2+2 s}+\delta^{-4} N^{-2-2 s}+\delta^{-2} N^{-2 s}\right)\|\nabla v\|_{H^{s}\left(\mathbb{R}^{n}\right)}^{2} .
$$

Finally, combining (30) with (26) and choosing $N^{-1}=\delta=\epsilon$, we conclude

$$
\left\|\operatorname{dist}\left(\nabla \tilde{v}_{N}, \tilde{K}\right)\right\|_{L^{2}(\Omega)}^{2}+C \epsilon^{2}\left\|\nabla^{2} \tilde{v}_{N}\right\|_{L^{2}(\Omega)}^{2} \leq C \epsilon^{2 s}\|\nabla v\|_{H^{s}\left(\mathbb{R}^{n}\right)}^{2} .
$$

As $\|\nabla v\|_{H^{s}\left(\mathbb{R}^{n}\right)}^{2}$ is bounded independently of $\epsilon$, this concludes the argument.

In order to illustrate that the choice of our specific regularisation $\tilde{v}_{N}$ of $v$ was not so important, we present a second, convolution-based proof of Theorem 1:

Proof of Theorem 1 by convolution. Let $\varphi \in \mathcal{S}$ with $\|\varphi\|_{L^{\infty}}=1, \int \varphi=1$ and let $\varphi_{\mu}(x):=\mu^{n} \varphi(\mu x)$. Denoting $\tilde{v}_{\mu}(x):=\eta_{\delta}(x) v_{\mu}(x)$ with $v_{\mu}(x)=\left(\varphi_{\mu} * v\right)(x)$ and $v(x)=u(x)-M x-b$, we argue similarly as before: As $\nabla v \in \tilde{K}$

$$
\begin{aligned}
\left\|\operatorname{dist}\left(\nabla \tilde{v}_{\mu}, \tilde{K}\right)\right\|_{L^{2}(\Omega)}^{2} \leq & C\left(\left\|\left(1-\eta_{\delta}\right) \nabla v\right\|_{L^{2}(\Omega)}^{2}+\left\|\nabla v-\nabla v_{\mu}\right\|_{L^{2}(\Omega)}^{2}\right. \\
& \left.+\left\|v_{\mu} \nabla \eta_{\delta}\right\|_{L^{2}(\Omega)}^{2}\right) .
\end{aligned}
$$

We bound the three right hand side contributions in (32) separately. First by Poincaré's inequality,

$$
\left\|\left(1-\varphi_{\delta}\right) \nabla v\right\|_{L^{2}(\Omega)}^{2} \leq\|\nabla v\|_{L^{2}\left(\Omega \backslash \Omega_{2 \delta}\right)}^{2} \leq C \delta^{2 s}\|\nabla v\|_{H^{s}\left(\mathbb{R}^{n}\right)}^{2} .
$$

Secondly, by scaling,

$$
\begin{aligned}
\left\|\nabla v-\nabla v_{\mu}\right\|_{L^{2}\left(\mathbb{R}^{n}\right)}^{2} & =\left\|\nabla v-\varphi_{\mu} * \nabla v\right\|_{L^{2}\left(\mathbb{R}^{n}\right)}^{2}=\left\||\cdot|^{-s}\left(1-\mathcal{F} \varphi_{\mu}\right)|\cdot|{ }^{s} \mathcal{F}(\nabla v)\right\|_{L^{2}\left(\mathbb{R}^{n}\right)}^{2} \\
& \leq\left\||\cdot|^{-s}\left(1-\mathcal{F} \varphi_{\mu}\right)\right\|_{L^{\infty}\left(\mathbb{R}^{n}\right)}^{2}\left\||\nabla|^{s} \nabla v\right\|_{L^{2}\left(\mathbb{R}^{n}\right)}^{2} \\
& \leq C \mu^{2 s}\|v\|_{H^{s}\left(\mathbb{R}^{n}\right)}^{2} .
\end{aligned}
$$


In order to bound $\left\||\cdot|^{-s}\left(1-\mathcal{F} \varphi_{\mu}\right)\right\|_{L^{\infty}\left(\mathbb{R}^{n}\right)}$, we used that $|k|^{-s}\left(1-\left(\mathcal{F} \varphi_{\mu}\right)(k)\right)=$ $\mu^{s}|\xi|^{-s}(1-(\mathcal{F} \varphi)(\xi))$ as well as the fact that $\mathcal{F}(\varphi)(0)=1$ (by our normalisation) and the regularity of $\mathcal{F} \varphi$. For the third contribution we estimate by combining the support condition for $\eta_{\delta}$ with scaling:

$$
\begin{aligned}
\left\|v_{\mu} \nabla \eta_{\delta}\right\|_{L^{2}\left(\mathbb{R}^{n}\right)}^{2} & \leq C \delta^{-2}\left\|v_{\mu}\right\|_{L^{2}\left(\mathbb{R}^{n}\right)}^{2} \leq C \delta^{-2}\left\|\left(|\cdot|^{-(1+s)} \mathcal{F} v\right)\left(|\cdot|^{1+s} \mathcal{F} \varphi_{\mu}\right)\right\|_{L^{2}\left(\mathbb{R}^{n}\right)}^{2} \\
& \leq C \delta^{-2}\left\||\cdot|^{1+s} \mathcal{F} \varphi_{\mu}\right\|_{L^{2}\left(\mathbb{R}^{n}\right)}^{2}\left\||\cdot|^{-(1+s)} \mathcal{F} v\right\|_{L^{2}\left(\mathbb{R}^{n}\right)}^{2} \\
& \leq C \delta^{-2} \mu^{2+2 s}\|v\|_{H^{s}\left(\mathbb{R}^{n}\right)}^{2} .
\end{aligned}
$$

Hence, inserting (33)-(35) into (32) entails

$$
\left\|\operatorname{dist}\left(\nabla \tilde{v}_{\mu}, \tilde{K}\right)\right\|_{L^{2}(\Omega)}^{2} \leq C\left(\delta^{2 s}+\mu^{2 s}+\delta^{-2} \mu^{2+2 s}\right)\|\nabla v\|_{H^{s}\left(\mathbb{R}^{n}\right)}^{2} .
$$

Finally, we bound the surface energy contribution by applying Poincaré's inequality and scaling:

$$
\begin{aligned}
\left\|\nabla^{2} \tilde{v}_{\mu}\right\|_{L^{2}\left(\mathbb{R}^{n}\right)}^{2} \leq & C\left(\left\|\nabla^{2} v_{\mu}\right\|_{L^{2}\left(\mathbb{R}^{n}\right)}^{2}+\left\|v_{\mu} \nabla^{2} \eta_{\delta}\right\|_{L^{2}\left(\mathbb{R}^{n}\right)}^{2}+\left\|\nabla \eta_{\delta} \cdot \nabla v_{\mu}\right\|_{L^{2}\left(\mathbb{R}^{n}\right)}^{2}\right) \\
\leq & C\left(\mu^{-2+2 s}\|\nabla v\|_{H^{s}\left(\mathbb{R}^{n}\right)}^{2}+\delta^{-4}\left\|v_{\mu}\right\|_{L^{2}\left(\Omega \backslash \Omega_{2 \delta}\right)}^{2}+\delta^{-2}\left\|\nabla v_{\mu}\right\|_{L^{2}\left(\mathbb{R}^{n}\right)}^{2}\right) \\
\leq & C\left(\mu^{-2+2 s}\|\nabla v\|_{H^{s}\left(\mathbb{R}^{n}\right)}^{2}+\delta^{-2+2 s}\left\|v_{\mu}\right\|_{H^{s}\left(\Omega \backslash \Omega_{2 \delta}\right)}^{2}\right. \\
& \left.+\delta^{-2} \mu^{2 s}\left\|\nabla v_{\mu}\right\|_{H^{s}\left(\mathbb{R}^{n}\right)}^{2}\right) .
\end{aligned}
$$

Combining (36) and (37) and choosing $\delta \sim \mu$, thus entails

$$
\left\|\operatorname{dist}\left(\nabla \tilde{v}_{\mu}, \tilde{K}\right)\right\|_{L^{2}(\Omega)}^{2}+\epsilon^{2}\left\|\nabla^{2} \tilde{v}_{\mu}\right\|_{L^{2}(\Omega)}^{2} \leq C\left(\delta^{2 s}+\epsilon^{2} \delta^{2 s-2}\right)\|\nabla v\|_{H^{s}\left(\mathbb{R}^{n}\right)}^{2} .
$$

Optimising and choosing $\delta \sim \epsilon$ yields the desired claim.

Remark 2.1. We remark that the above argument is not restricted to the setting of the geometrically nonlinear theory of elasticity but also works with minor modifications in the geometrically linearised setting. Indeed, setting $e(M):=\frac{1}{2}\left(M+M^{t}\right)$ for $M \in \mathbb{R}^{n \times n}$, if one was to consider a differential inclusion of the form

$$
\begin{aligned}
e(\nabla u) & \in K \text { in } \Omega, \\
\nabla u & =M \text { on } \partial \Omega,
\end{aligned}
$$

for some $u$ with the property that $u-M x-b \in H^{1+s}\left(\mathbb{R}^{n}\right)$ for some $b \in \mathbb{R}^{n}$ and $M \in \mathbb{R}^{n \times n}$, then one would also obtain that for some constant $C>0$ which might depend on $n, \Omega, M, K$

$$
\bar{E}_{\epsilon}:=\min _{\nabla u=M \text { on } \mathbb{R}^{n} \backslash \bar{\Omega}} \int_{\Omega} \operatorname{dist}^{2}(e(\nabla u), K) d x+\epsilon^{2} \int_{\Omega}|\nabla e(\nabla u)|^{2} d x \leq C \epsilon^{2 s} .
$$

In order to infer this, we again pass from $u$ to $v(x)=u(x)-M x-b$. The energy then transforms into

$$
\widetilde{E}_{\epsilon}:=\min _{\nabla v=0 \text { on } \mathbb{R}^{n} \backslash \bar{\Omega}} \int_{\mathbb{R}^{n}} \operatorname{dist}^{2}(e(\nabla v), \tilde{K}) d x+\epsilon^{2} \int_{\mathbb{R}^{n}}|\nabla e(\nabla v)|^{2} d x,
$$

where $\tilde{K}:=K-e(M)$. Recalling the pointwise inequality

$$
|a \otimes k+k \otimes a|^{2} \geq|a \otimes k|^{2},
$$

and arguing on the Fourier side, we infer the following Korn-type estimate

$$
\int_{\mathbb{R}^{n}}|\nabla e(\nabla v)|^{2} d x \geq c \int_{\mathbb{R}^{n}}\left|\nabla^{2} v\right|^{2} d x .
$$


In order to show the pointwise inequality (38), by finite dimensionality, without loss of generality, we take the matrix norm to be the $l^{2}$ norm, and compute explicitly:

$$
\begin{aligned}
\|a \otimes k\|_{2}^{2} & =\sum_{i, j}\left|a_{i} k_{j}\right|^{2}=\sum_{i}\left|a_{i}\right|^{2} \sum_{j}\left|k_{j}\right|^{2}=|a|^{2}|k|^{2}, \\
\|a \otimes k+k \otimes a\|_{2}^{2} & =\sum_{i, j}\left|a_{i} k_{j}+a_{j} k_{i}\right|^{2}=\sum_{i, j}\left|a_{i} k_{j}\right|^{2}+\left|a_{j} k_{i}\right|^{2}+2 a_{i} k_{j} a_{j} k_{i} \\
& =2|a|^{2}|k|^{2}+2(a \cdot k)^{2} \geq 2|a|^{2}|k|^{2}=2\|a \otimes k\|_{2}^{2} .
\end{aligned}
$$

Thus, in order to deduce the desired estimate for $\bar{E}_{\epsilon}$, it suffices to prove that

$$
\widetilde{E}_{\epsilon}:=\min _{\nabla v=0 \text { on } \mathbb{R}^{n} \backslash \bar{\Omega}} \int_{\mathbb{R}^{n}} \operatorname{dist}^{2}(e(\nabla v), \tilde{K}) d x+\epsilon^{2} \int_{\mathbb{R}^{n}}\left|\nabla^{2} v\right|^{2} d x \leq C \epsilon^{2 s}
$$

With this reduction in hand, all the arguments from the proof for the geometrically nonlinear setting also directly transfer to the geometrically linearised setting. This remains valid for norms in which a Korn-type estimate like (39) holds (but excludes spaces like $L^{1}$ in which these Calderón-Zygmund type arguments fail).

2.3. Variations on Theorem 1. Instead of working in $L^{2}$ based function spaces, we can also work with a Littlewood-Paley decomposition and the associated Bernstein estimates in other function spaces, i.e. for different energy functionals. We stress that it is physically significant to be able to work in different function spaces, since the precise form of surface energies is not known in general. Hence, it is important to obtain a robust mechanism which provides results in a large class of possible surface energy models ranging from diffuse models as in Theorem 1 to sharp interface models as in Proposition 2.2.

Consider for instance the (sharp interface) energy functional

$$
E_{\epsilon, M, 1}=\min _{\nabla u=M \text { a.e. in } \mathbb{R}^{n} \backslash \bar{\Omega}}\left\{\int_{\Omega} \operatorname{dist}^{2}(\nabla u, K) d x+\epsilon\left|\nabla^{2} u\right|(\Omega)\right\},
$$

where $\left|\nabla^{2} u\right|(\Omega)$ denotes the total variation of the distributional derivative of $\nabla u$.

As in Theorem 1 we obtain the following bound for possible convex integration solutions:

Proposition 2.2. Let $E_{\epsilon, M, 1}$ be the energy from (40). Assume that there exists a solution $u$ to the differential inclusion (7) such that $v(x):=u(x)-M x-b \in$ $B_{1,1}^{1+s}\left(\mathbb{R}^{n}\right)$ for some $b \in \mathbb{R}^{n}, s \in \mathbb{R}$ with $\operatorname{supp}(v) \subset \bar{\Omega}$ and $\nabla v \in L^{\infty}\left(\mathbb{R}^{n}\right)$. Then there exists a constant $C=C(M, \Omega, n, s)>1$ such that

$$
E_{\epsilon, M, 1} \leq C \epsilon^{s} \text {. }
$$

Here $B_{1,1}^{1+s}\left(\mathbb{R}^{n}\right)$ denotes the standard Besov space (defined for instance through a suitable Littlewood-Paley decomposition, see Section 2.1 and [BCD11]). Loosely speaking the proposition states, that on a Besov scale, for convex integration solutions we can at most have $\nabla u \in\left(B_{1,1}^{s}\right)_{l o c}\left(\mathbb{R}^{n}\right)$, if there is a lower bound $E_{\epsilon, M, 1} \geq C \epsilon^{s}$.

As in the case of Theorem 1, it is possible to complement also the result of Proposition 2.2 by a model-independent lower bound: ${ }^{2}$

\footnotetext{
${ }^{2}$ Again, we stress that complementing our result by a model independent lower bound had been suggested to us by one of the referees.
} 
Proposition 2.3. Let $\Omega \subset \mathbb{R}^{n}$ be a $C^{2}$ regular domain and let

$$
\mathcal{E}_{1, \epsilon}(u):=\int_{\Omega} \operatorname{dist}^{2}(\nabla u, K) d x+\epsilon\left|\nabla^{2} u\right|\left(\mathbb{R}^{n}\right),
$$

where $K \subset \mathbb{R}^{n \times n}$ is a bounded set. Then for all $u: \mathbb{R}^{n} \rightarrow \mathbb{R}^{n}$ with $\nabla u=M$ in $\mathbb{R}^{n} \backslash \bar{\Omega}$ there exists a constant $C=C(\Omega, n, K)>0$ such that

$$
\mathcal{E}_{1, \epsilon}(u) \geq C \min \left(\operatorname{dist}(M, K), \operatorname{dist}(M, K)^{2}\right) \epsilon .
$$

We postpone the proof of Proposition 2.3 to Section 2.4 and first discuss the proof of Proposition 2.2.

Proof of Proposition 2.2. Step 1: Preliminaries. As a first preliminary step, we again switch to the situation where

$$
v(x)=u(x)-M x-b,
$$

and $b \in \mathbb{R}^{n}$ is chosen such that $v \in B_{1,1}^{1+s}\left(\mathbb{R}^{n}\right)$ and $\operatorname{supp}(v) \subset \bar{\Omega}$. The modified energy wells turn into $\tilde{K}:=K-M$, and the energy (40) becomes

$$
\tilde{E}_{\epsilon, M, 1}:=\min _{\nabla v=0 \text { a.e. in } \mathbb{R}^{n} \backslash \bar{\Omega}}\left\{\|\operatorname{dist}(\nabla v, \tilde{K})\|_{L^{2}(\Omega)}^{2}+\epsilon\left|\nabla^{2} v\right|(\Omega)\right\} .
$$

Given a convex integration solution $u$ of (7) or $v$ of (17), we again consider the functions

$$
\tilde{v}_{N}(x):=\eta_{\delta}(x) v_{<N}(x),
$$

where $\eta_{\delta}$ is a smooth, positive cut-off function which is equal to one in $\Omega_{2 \delta}:=\{x \in$ $\Omega: \operatorname{dist}(x, \partial \Omega)>2 \delta\}$, which vanishes outside of $\Omega_{\delta}:=\{x \in \Omega: \operatorname{dist}(x, \partial \Omega)>\delta\}$ and which satisfies

$$
\left|\nabla \eta_{\delta}\right| \leq \frac{C}{\delta},\left|\nabla^{2} \eta_{\delta}\right| \leq \frac{C}{\delta^{2}}
$$

The value of the parameter $\delta$ will be determined in the sequel. Using Bernstein type inequalities [BCD11] and the embeddings $B_{1,1}^{0}\left(\mathbb{R}^{n}\right) \subset L^{1}\left(\mathbb{R}^{n}\right)$ (see Theorem 2.41 in $[\mathrm{BCD} 11])$ and $W^{1,1}(\Omega) \subset B V(\Omega)$, for $v$ with $\nabla v \in L^{\infty}\left(\mathbb{R}^{n}\right) \cap B_{1,1}^{s}\left(\mathbb{R}^{n}\right)$ we deduce

$$
\begin{aligned}
\left|\nabla(\nabla v)_{<N}\right|(\Omega) & \leq\left\|(\nabla v)_{<N}\right\|_{\dot{W}^{1,1}\left(\mathbb{R}^{n}\right)} \leq C\left\|\left(\nabla^{2} v\right)_{<N}\right\|_{B_{1,1}^{0}\left(\mathbb{R}^{n}\right)} \\
& \leq C N^{1-s}\|\nabla v\|_{B_{1,1}^{s}\left(\mathbb{R}^{n}\right)}, \\
\left\|(\nabla v)_{\geq N}\right\|_{L^{1}(\Omega)} & \leq\left\|(\nabla v)_{\geq N}\right\|_{L^{1}\left(\mathbb{R}^{n}\right)} \leq C\left\|(\nabla v)_{\geq N}\right\|_{B_{1,1}^{0}\left(\mathbb{R}^{n}\right)} \leq C N^{-s}\|\nabla v\|_{B_{1,1}^{s}\left(\mathbb{R}^{n}\right)} .
\end{aligned}
$$

Step 2: Bulk energy estimate. With the observations from Step 1 in hand, using that

$$
\nabla \tilde{v}_{N}=\eta_{\delta} \nabla v_{<N}+v_{<N} \nabla \eta_{\delta}
$$

and using the properties of the cut-off $\eta_{\delta}$ (see (42)), we estimate as follows:

$$
\begin{aligned}
& \left\|\operatorname{dist}\left(\nabla \tilde{v}_{N}, \tilde{K}\right)\right\|_{L^{2}(\Omega)}^{2} \leq 2\left\|\operatorname{dist}\left(\eta_{\delta} \nabla v_{<N}, \tilde{K}\right)\right\|_{L^{2}(\Omega)}^{2}+2\left\|v_{<N} \nabla \eta_{\delta}\right\|_{L^{2}(\Omega)}^{2} \\
& \leq 2\left\|\operatorname{dist}\left(\eta_{\delta} \nabla v_{<N}, \tilde{K}\right)\right\|_{L^{2}(\Omega)}^{2}+2\left\|v_{<N} \nabla \eta_{\delta}\right\|_{L^{\infty}\left(\mathbb{R}^{n}\right)}\left\|v_{<N} \nabla \eta_{\delta}\right\|_{L^{1}\left(\mathbb{R}^{n}\right)}
\end{aligned}
$$

We estimate the terms in (44) separately and begin by bounding the second right hand side contribution: As a first step towards this we note that

$$
\left\|v_{<N} \nabla \eta_{\delta}\right\|_{L^{q}\left(\mathbb{R}^{n}\right)} \leq C\left(\left\|v \nabla \eta_{\delta}\right\|_{L^{q}\left(\mathbb{R}^{n}\right)}+\left\|v_{\geq N} \nabla \eta_{\delta}\right\|_{L^{q}\left(\mathbb{R}^{n}\right)}\right)
$$


for $q \in\{1, \infty\}$. By Bernstein estimates and (13), we control the second term in (45) for $q=\infty$ as follows

$$
\begin{aligned}
\left\|v_{\geq N} \nabla \eta_{\delta}\right\|_{L^{\infty}\left(\mathbb{R}^{n}\right)} & \leq C \delta^{-1}\left\|v_{\geq N}\right\|_{L^{\infty}\left(\mathbb{R}^{n}\right)} \leq C \delta^{-1} N^{-1}\left\|\nabla v_{\geq N}\right\|_{L^{\infty}\left(\mathbb{R}^{n}\right)} \\
& \leq C \delta^{-1} N^{-1}\|\nabla v\|_{L^{\infty}\left(\mathbb{R}^{n}\right)}
\end{aligned}
$$

For $q=1$ and also by (13) and by Bernstein estimates, see (20), we infer

$$
\begin{aligned}
& \left\|v_{\geq N} \nabla \eta_{\delta}\right\|_{L^{1}\left(\mathbb{R}^{n}\right)} \leq\left\|\nabla \eta_{\delta}\right\|_{L^{\infty}\left(\mathbb{R}^{n}\right)}\left\|v_{\geq N}\right\|_{L^{1}\left(\mathbb{R}^{n}\right)} \\
& \leq C \frac{1}{\delta} \frac{1}{N^{1+s}}\|\nabla v\|_{B_{1,1}^{s}\left(\mathbb{R}^{n}\right)} .
\end{aligned}
$$

The respective first terms on the right hand side of (45) are controlled by invoking the (fractional) Poincaré inequality (see Appendix A)

$$
\begin{aligned}
\left\|v \nabla \eta_{\delta}\right\|_{L^{\infty}\left(\mathbb{R}^{n}\right)} & \leq C \delta^{-1}\|v\|_{L^{\infty}\left(\Omega_{\delta} \backslash \Omega_{2 \delta}\right)} \leq C \delta^{-1} \delta\|\nabla v\|_{L^{\infty}\left(\Omega \backslash \Omega_{2 \delta}\right)}, \\
\left\|v \nabla \eta_{\delta}\right\|_{L^{1}\left(\mathbb{R}^{n}\right)} & \leq C \delta^{-1}\|v\|_{L^{1}\left(\Omega_{\delta} \backslash \Omega_{2 \delta}\right)} \leq C \delta^{-1} \delta\|\nabla v\|_{L^{1}\left(\Omega \backslash \Omega_{2 \delta}\right)} \leq C \delta^{s}\|\nabla v\|_{B_{1,1}^{s}\left(\mathbb{R}^{n}\right)} .
\end{aligned}
$$

Combining (46)-(48) thus yields

$$
\begin{aligned}
& \left\|v_{<N} \nabla \eta_{\delta}\right\|_{L^{\infty}\left(\mathbb{R}^{n}\right)}\left\|v_{<N} \nabla \eta_{\delta}\right\|_{L^{1}\left(\mathbb{R}^{n}\right)} \\
& \leq C\left(\delta^{-2} N^{-2-s}+\delta^{s}+N^{-1-s} \delta^{-1}+\delta^{s}\right)\|\nabla v\|_{L^{\infty}\left(\mathbb{R}^{n}\right)}\|\nabla v\|_{B_{1,1}^{s}\left(\mathbb{R}^{n}\right)}
\end{aligned}
$$

Next we estimate the first term in (44): Again by Poincaré, Bernstein, the estimate in (13) and further by $\nabla v \in \tilde{K}$ we observe

$$
\begin{aligned}
& \left\|\operatorname{dist}\left(\eta_{\delta} \nabla v_{<N}, \tilde{K}\right)\right\|_{L^{2}(\Omega)}^{2} \leq C\left\|\operatorname{dist}\left(\eta_{\delta} \nabla v_{<N}, \tilde{K}\right)\right\|_{L^{1}(\Omega)}\left(\left\|\nabla v_{<N}\right\|_{L^{\infty}(\Omega)}+C_{\tilde{K}}\right) \\
& \leq C\left(\left\|\operatorname{dist}\left(\eta_{\delta} \nabla v, \tilde{K}\right)\right\|_{L^{1}(\Omega)}+\left\|\nabla v-\nabla v_{<N}\right\|_{L^{1}(\Omega)}\right)\left(\left\|\nabla v_{<N}\right\|_{L^{\infty}\left(\mathbb{R}^{n}\right)}+C_{\tilde{K}}\right) \\
& \leq C\left(\|\operatorname{dist}(\nabla v, \tilde{K})\|_{L^{1}(\Omega)}+\left\|\left(1-\eta_{\delta}\right) \nabla v\right\|_{L^{1}(\Omega)}+\left\|\nabla v-\nabla v_{<N}\right\|_{L^{1}\left(\mathbb{R}^{n}\right)}\right) \\
& \quad \times\left(\|\nabla v\|_{L^{\infty}\left(\mathbb{R}^{n}\right)}+C_{\tilde{K}}\right) \\
& \leq C\left(\delta^{s}+N^{-s}\right)\|\nabla v\|_{B_{1,1}^{s}\left(\mathbb{R}^{n}\right)}\left(\|\nabla v\|_{L^{\infty}\left(\mathbb{R}^{n}\right)}+C_{\tilde{K}}\right) .
\end{aligned}
$$

Here, the constant $C_{\tilde{K}}$ is given by $\operatorname{dist}(0, \tilde{K})=\operatorname{dist}(M, K)$. Combining (44) with (49) and (50), we infer for the elastic energy

$$
\begin{aligned}
\left\|\operatorname{dist}\left(\nabla \tilde{v}_{N}, \tilde{K}\right)\right\|_{L^{2}(\Omega)}^{2} \leq & C\left(\delta^{s}+N^{-s}+\delta^{-1} N^{-1-s}+\delta^{-2} N^{-2-s}\right) \\
& \times\left(\|\nabla v\|_{L^{\infty}\left(\mathbb{R}^{n}\right)}+C_{\tilde{K}}\right) \times\left(\|\nabla v\|_{L^{\infty}\left(\mathbb{R}^{n}\right)}+\|\nabla v\|_{B_{1,1}^{s}\left(\mathbb{R}^{n}\right)}\right) .
\end{aligned}
$$


Step 3: Estimate of the surface energy and conclusion. For the surface energy we estimate

$$
\begin{aligned}
\left|\nabla^{2} \tilde{v}_{N}\right|(\Omega) \leq & \left\|\nabla^{2} v_{<N}\right\|_{L^{1}(\Omega)}+2\left\|\nabla \eta_{\delta} \nabla v_{<N}\right\|_{L^{1}(\Omega)}+\left\|v_{<N} \nabla^{2} \eta_{\delta}\right\|_{L^{1}(\Omega)} \\
\leq & \left\|\nabla^{2} v_{<N}\right\|_{L^{1}(\Omega)}+2\left\|\nabla \eta_{\delta} \nabla v_{\geq N}\right\|_{L^{1}\left(\mathbb{R}^{n}\right)}+2\left\|\nabla \eta_{\delta} \nabla v\right\|_{L^{1}\left(\mathbb{R}^{n}\right)} \\
& +\left\|v_{\geq N} \nabla^{2} \eta_{\delta}\right\|_{L^{1}\left(\mathbb{R}^{n}\right)}+2\left\|v \nabla^{2} \eta_{\delta}\right\|_{L^{1}\left(\mathbb{R}^{n}\right)} \\
\leq & C\left(\left\|\nabla^{2} v_{<N}\right\|_{L^{1}(\Omega)}+\frac{1}{\delta}\left\|\nabla v_{\geq N}\right\|_{L^{1}\left(\mathbb{R}^{n}\right)}+\frac{1}{\delta^{2}}\left\|v_{\geq N}\right\|_{L^{1}\left(\mathbb{R}^{n}\right)}\right. \\
& \left.+\frac{1}{\delta}\|\nabla v\|_{L^{1}\left(\Omega \backslash \Omega_{2 \delta}\right)}+\frac{1}{\delta^{2}}\|v\|_{L^{1}\left(\Omega \backslash \Omega_{2 \delta}\right)}\right) \\
\leq & C\left(N^{1-s}\|\nabla v\|_{B_{1,1}^{s}\left(\mathbb{R}^{n}\right)}+\frac{2}{\delta N^{s}}\|\nabla v\|_{B_{1,1}^{s}\left(\mathbb{R}^{n}\right)}+\frac{1}{\delta^{2} N^{1+s}}\|\nabla v\|_{B_{1,1}^{s}\left(\mathbb{R}^{n}\right)}\right. \\
& \left.+\frac{2 \delta^{s}}{\delta}\|\nabla v\|_{B_{1,1}^{s}\left(\mathbb{R}^{n}\right)}+\frac{\delta^{1+s}}{\delta^{2}}\|\nabla v\|_{B_{1,1}^{s}\left(\mathbb{R}^{n}\right)}\right) .
\end{aligned}
$$

Here we have used some of the Bernstein type estimates which were stated in (43) in combination with a fractional Poincaré inequality (see Appendix A). Combining (51) and (52) and choosing $N=\delta^{-1}$ yields

$$
\begin{aligned}
\tilde{E}_{\epsilon, M, 1} & \leq\left\|\operatorname{dist}\left(\nabla \tilde{v}_{N}, K\right)\right\|_{L^{2}(\Omega)}^{2}+\epsilon\left|\nabla^{2} \tilde{v}_{N}\right|(\Omega) \\
& \leq C\left(\|\nabla v\|_{L^{\infty}\left(\mathbb{R}^{n}\right)},\|\nabla v\|_{B_{1,1}^{s}\left(\mathbb{R}^{n}\right)},|\Omega|, C_{\tilde{K}}\right)\left(N^{s}+\epsilon N^{1-s}\right) .
\end{aligned}
$$

Choosing $N \sim \epsilon^{-1}$, we hence obtain

$$
\tilde{E}_{\epsilon, M, 1} \leq C\left(\|\nabla v\|_{L^{\infty}\left(\mathbb{R}^{n}\right)},\|\nabla v\|_{B_{1,1}^{s}\left(\mathbb{R}^{n}\right)}\right) \epsilon^{s} .
$$

Returning from $\tilde{E}_{\epsilon, M, 1}$ to $E_{\epsilon, M, 1}$ therefore proves the claim.

More generally, the previous situation can be studied with regularisations in the Sobolev spaces $W^{s, p}$. In this setting we have the following result:

Proposition 2.4. Let $1<p \leq 2$ and denote by $E_{\epsilon, M, p}$ the minimal energy

$$
E_{\epsilon, M, p}=\min _{\nabla u=M \text { a.e. in } \mathbb{R}^{n} \backslash \bar{\Omega}}\left\{\int_{\Omega} \operatorname{dist}^{2}(\nabla u, K) d x+\epsilon^{p}\left\|\nabla^{2} u\right\|_{L^{p}(\Omega)}^{p}\right\} .
$$

Assume that there exists a solution $u$ to the differential inclusion (7) such that $v(x):=u(x)-M x-b \in W^{s+1, p}\left(\mathbb{R}^{n}\right)$ for some $b \in \mathbb{R}^{n}, s \in \mathbb{R}$ with $\operatorname{supp}(v) \subset \bar{\Omega}$ and $\nabla v \in L^{\infty}\left(\mathbb{R}^{n}\right)$. Then there exists a constant $C=C(M, \Omega, n, s)>1$ such that

$$
E_{\epsilon, M, p} \leq C \epsilon^{s p} \text {. }
$$

In other words, the scaling behaviour yields an upper bound on the regularity of convex integration solutions, i.e. on a Sobolev $W_{l o c}^{s, p}$ scale, for convex integration solutions we can at most have $\nabla u \in W_{l o c}^{\frac{2 \mu}{p}, p}\left(\mathbb{R}^{n}\right) \cap L^{\infty}\left(\mathbb{R}^{n}\right)$, if it holds that $E_{\epsilon, M, p} \geq$ $C \epsilon^{s p}$.

In analogy to the results of Theorem 2 and Proposition 2.3, it is possible to also derive a corresponding lower bound in this model:

Proposition 2.5. Let $\Omega \subset \mathbb{R}^{n}$ be a $C^{2}$ regular domain and let

$$
\mathcal{E}_{p, \epsilon}(u):=\int_{\Omega} \operatorname{dist}^{2}(\nabla u, K) d x+\epsilon^{p}\left\|\nabla^{2} u\right\|_{L^{p}(\Omega)}^{p},
$$

where $K \subset \mathbb{R}^{n \times n}$ is a bounded set. Then for all $u: \mathbb{R}^{n} \rightarrow \mathbb{R}^{n}$ with $\nabla u=M$ in $\mathbb{R}^{n} \backslash \bar{\Omega}$ there exists a constant $C=C(\Omega, n, K, p)>0$ such that

$$
\mathcal{E}_{p, \epsilon}(u) \geq C \min \left(\operatorname{dist}(M, K)^{2-p}, \operatorname{dist}(M, K)^{2}\right) \epsilon .
$$


We postpone the proof of Proposition 2.5 to Section 2.4.

The proof of Proposition 2.4 proceeds as in the $B V$ setting by using Bernstein type estimates.

Proof of Proposition 2.4. Step 1: Preliminaries. As in the previous proofs, we reduce to the shifted problem by setting $v(x)=u(x)-M x-b$ for which we assume that for some $b \in \mathbb{R}^{n}$ we have $v \in W^{s, p}\left(\mathbb{R}^{n}\right)$ and $\operatorname{supp}(v) \subset \bar{\Omega}$. Hence, if $u$ is a solution to (7), then $v$ is a solution to (17). The correspondingly modified energy reads

$$
\tilde{E}_{\epsilon, M, p}=\min _{\nabla v=0 \text { a.e. in } \mathbb{R}^{n} \backslash \bar{\Omega}}\left\{\int_{\Omega} \operatorname{dist}^{2}(\nabla v, \tilde{K}) d x+\epsilon^{p}\left\|\nabla^{2} v\right\|_{L^{p}(\Omega)}^{p}\right\} .
$$

As in the proof of Proposition 2.2, we use the standard Littlewood-Paley decomposition to define

$$
\tilde{v}_{N}(x):=\eta_{\delta}(x) v_{<N}(x) .
$$

Step 1: Bulk estimate. Throughout the proof we choose $N=\delta^{-1}$. We thus estimate

$$
\begin{aligned}
\left\|\operatorname{dist}\left(\nabla \tilde{v}_{N}, \tilde{K}\right)\right\|_{L^{2}(\Omega)}^{2} \leq & C\left(\left\|\operatorname{dist}\left(\eta_{\delta} \nabla v_{<N}, \tilde{K}\right)\right\|_{L^{2}(\Omega)}^{2}+\left\|v_{<N} \nabla \eta_{\delta}\right\|_{L^{2}(\Omega)}^{2}\right) \\
\leq & C\left(\left\|\operatorname{dist}\left(\eta_{\delta} \nabla v_{<N}, \tilde{K}\right)\right\|_{L^{2}(\Omega)}^{2}\right. \\
& \left.+\left\|v_{<N} \nabla \eta_{\delta}\right\|_{L^{p}\left(\mathbb{R}^{n}\right)}^{p}\left\|v_{<N} \nabla \eta_{\delta}\right\|_{L^{\infty}\left(\mathbb{R}^{n}\right)}^{2-p}\right) \\
\leq & C\left(\left\|\operatorname{dist}\left(\eta_{\delta} \nabla v_{<N}, \tilde{K}\right)\right\|_{L^{2}(\Omega)}^{2}\right. \\
& \left.+\delta^{s p}\|\nabla v\|_{W^{s, p}\left(\mathbb{R}^{n}\right)}^{p}\|\nabla v\|_{L^{\infty}(\Omega)}^{2-p}\right),
\end{aligned}
$$

where we used similar estimates as in (45)-(47) in the proof of Proposition 2.2. Furthermore, (using that $\delta=N^{-1}$ )

$$
\begin{aligned}
\left\|\operatorname{dist}\left(\eta_{\delta} \nabla v_{<N}, \tilde{K}\right)\right\|_{L^{2}(\Omega)}^{2} \leq & C\left(\left\|\operatorname{dist}\left(\nabla v_{<N}, \tilde{K}\right)\right\|_{L^{p}(\Omega)}^{p}+\left\|\left(1-\eta_{\delta}\right) \nabla v_{<N}\right\|_{L^{p}(\Omega)}^{p}\right) \times \\
& \times\left(\left\|\operatorname{dist}\left(\nabla v_{<N}, \tilde{K}\right)\right\|_{L^{\infty}(\Omega)}^{2-p}+\left\|\left(1-\eta_{\delta}\right) \nabla v_{<N}\right\|_{L^{\infty}(\Omega)}^{2-p}\right) \\
\leq & C\left(\|\operatorname{dist}(\nabla v, \tilde{K})\|_{L^{p}(\Omega)}^{p}+\left\|\left(1-\eta_{\delta}\right) \nabla v\right\|_{L^{p}\left(\mathbb{R}^{n}\right)}^{p}\right. \\
& \left.+\left\|\left(1-\eta_{\delta}\right) \nabla v_{\geq N}\right\|_{L^{p}\left(\mathbb{R}^{n}\right)}^{p}+\left\|\nabla v-\nabla v_{<N}\right\|_{L^{p}\left(\mathbb{R}^{n}\right)}^{p}\right) \times \\
& \times\left(\|\nabla v\|_{L^{\infty}\left(\mathbb{R}^{n}\right)}^{2-p}+C_{\tilde{K}}\right) \\
\leq & C\left(\left\|\left(1-\eta_{\delta}\right) \nabla v\right\|_{L^{p}\left(\Omega \backslash \Omega_{2 \delta}\right)}^{p}+\left\|\nabla v_{\geq N}\right\|_{L^{p}\left(\mathbb{R}^{n}\right)}^{p}\right. \\
& \left.+\left\|\nabla v-\nabla v_{<N}\right\|_{L^{p}\left(\mathbb{R}^{n}\right)}^{p}\right)\left(\|\nabla v\|_{L^{\infty}\left(\mathbb{R}^{n}\right)}^{2-p}+C_{\tilde{K}}\right) \\
\leq & C\left(\delta^{p s}\|\nabla v\|_{W^{s, p}\left(\mathbb{R}^{n}\right)}^{p}+N^{p s}\|\nabla v\|_{W^{s, p}\left(\mathbb{R}^{n}\right)}^{p}\right) \times \\
& \times\left(\|\nabla u\|_{L^{\infty}\left(\mathbb{R}^{n}\right)}^{2-p}+C_{\tilde{K}}\right) \\
\leq & C \delta^{p s}\|\nabla v\|_{W^{s, p}\left(\mathbb{R}^{n}\right)}^{p}\left(\|\nabla v\|_{L^{\infty}\left(\mathbb{R}^{n}\right)}^{2-p}+C_{\tilde{K}}\right) .
\end{aligned}
$$

Here, $C_{\tilde{K}}=\operatorname{dist}(0, \tilde{K})^{2-p}$. Here we used Bernstein estimates, the fractional Poincaré estimate and the bounds from (13). Thus, combining (55), (56), the elastic energy is controlled by

$$
\left\|\operatorname{dist}\left(\nabla \tilde{v}_{N}, \tilde{K}\right)\right\|_{L^{2}(\Omega)}^{2} \leq C \delta^{p s}\|\nabla v\|_{W^{s, p}\left(\mathbb{R}^{n}\right)}^{p}\left(\|\nabla v\|_{L^{\infty}\left(\mathbb{R}^{n}\right)}^{2-p}+C_{\tilde{K}}\right) .
$$


Step 3: Surface energy bounds and conclusion. For the surface energy we estimate (by using Poincaré and Bernstein)

(58)

$$
\begin{aligned}
\left\|\nabla^{2} \tilde{v}_{N}\right\|_{L^{p}(\Omega)}^{p} \leq & C\left(\left\|\nabla^{2} v_{<N}\right\|_{L^{p}(\Omega)}^{p}+\left\|\nabla \eta_{\delta} \nabla v_{<N}\right\|_{L^{p}(\Omega)}^{p}+\left\|v_{<N} \nabla^{2} \eta_{\delta}\right\|_{L^{p}(\Omega)}^{p}\right) \\
\leq & C\left(\left\|\nabla^{2} v_{<N}\right\|_{L^{p}\left(\mathbb{R}^{n}\right)}^{p}+\left\|\nabla \eta_{\delta} \nabla v_{\geq N}\right\|_{L^{p}\left(\mathbb{R}^{n}\right)}^{p}+\left\|v_{\geq N} \nabla^{2} \eta_{\delta}\right\|_{L^{p}\left(\mathbb{R}^{n}\right)}^{p}\right. \\
& \left.+\left\|\nabla \eta_{\delta} \nabla v\right\|_{L^{p}\left(\mathbb{R}^{n}\right)}^{p}+\left\|v \nabla^{2} \eta_{\delta}\right\|_{L^{p}\left(\mathbb{R}^{n}\right)}^{p}\right) \\
\leq & C\left(N^{p-s p}+\delta^{-p} N^{-s p}+\delta^{-2 p} N^{-s p-p}\right. \\
& \left.\quad+\delta^{-p} \delta^{s p}+\delta^{-2 p} \delta^{p+s p}\right)\|\nabla v\|_{W^{s, p}\left(\mathbb{R}^{n}\right)}^{p} \\
\leq & C \delta^{-p+s p}\|\nabla v\|_{W^{s, p}\left(\mathbb{R}^{n}\right)}^{p},
\end{aligned}
$$

where we have again used $\delta=N^{-1}$. Hence, by combining (57) and (58), we infer

$$
\tilde{E}_{\epsilon, M, p} \leq C\left(\|\nabla v\|_{L^{\infty}(\Omega)},\|\nabla v\|_{W^{s, p}(\Omega)}, C_{\tilde{K}}\right)\left(\epsilon^{p} \delta^{-p+s p}+\delta^{s p}\right),
$$

for some $C \geq 1$. Further setting $\delta=\epsilon^{-1}$, we deduce the claimed upper bound.

Remark 2.6. Similarly as in [RZZ16] and in [Sic99], we have thus obtained that if a family of energy functionals is controlled, then the possible $W^{s, p}$ Sobolev regularity is determined in terms of the product sp and not by $s, p$ individually. Here the $L^{\infty}$ bound was crucial, as it allowed us to "break scaling".

2.4. Lower bounds. In this final section on rigidity, we present the proofs of Theorem 2 and of Propositions 2.3 and 2.5. These arguments are Modica-Mortolalike.

Proof of Theorem 2. Again we pass from $u$ to $v(x)=u(x)-M x-b$. We seek to prove that for all $v \in H_{0}^{2}(\Omega)$

$$
\tilde{\mathcal{E}}_{\epsilon}(v):=\int_{\Omega} \operatorname{dist}^{2}(\nabla v, \tilde{K})^{2} d x+\epsilon^{2} \int_{\Omega}\left|\nabla^{2} v\right|^{2} d x \geq C \operatorname{dist}^{2}(M, K) \epsilon,
$$

where $\tilde{K}=K-M$. Denoting by $d_{\partial \Omega}(x):=\operatorname{dist}(x, \partial \Omega)$ and applying the co-area formula yields

$$
\int_{\Omega} \operatorname{dist}^{2}(\nabla v, \tilde{K}) d x=\int_{0}^{\infty} \int_{\left\{d_{\partial \Omega}(x)=a\right\}} \operatorname{dist}^{2}(\nabla v, \tilde{K}) d \mathcal{H}^{n-1} d a .
$$

Furthermore, by Hardy's inequality (see for instance [OK90]) and the co-area formula

$$
\int_{\Omega}\left|\nabla^{2} v\right|^{2} d x \geq C \int_{\Omega} \frac{|\nabla v|^{2}}{d_{\partial \Omega}^{2}} d x=\int_{0}^{\infty} \int_{\left\{d_{\partial \Omega}=a\right\}} \frac{|\nabla v|^{2}}{a^{2}} d \mathcal{H}^{n-1} d a,
$$

where $d_{\partial \Omega}$ is the boundary distance function. Combining (59) with (60) therefore leads to

$$
\tilde{\mathcal{E}}_{\epsilon}(v) \geq \int_{0}^{\infty} \int_{\left\{d_{\partial \Omega}=a\right\}} \operatorname{dist}^{2}(\nabla v, \tilde{K})+\epsilon^{2} \frac{|\nabla v|^{2}}{a^{2}} d \mathcal{H}^{n-1} d a .
$$

Observing that by the triangle inequality

$$
\operatorname{dist}^{2}(\nabla v, \tilde{K})+|\nabla v|^{2} \geq \frac{1}{2} \operatorname{dist}^{2}(\tilde{K}, 0),
$$


and we obtain

$$
\begin{aligned}
\tilde{\mathcal{E}}_{\epsilon}(v) & \geq \int_{0}^{\epsilon} \int_{\left\{d_{\partial \Omega}=a\right\}} \operatorname{dist}^{2}(\nabla v, \tilde{K})+\epsilon^{2} \frac{|\nabla v|^{2}}{a^{2}} d \mathcal{H}^{n-1} d a \\
& \geq \int_{0}^{\epsilon} \int_{\left\{d_{\partial \Omega}=a\right\}} \operatorname{dist}^{2}(\nabla v, \tilde{K})+|\nabla v|^{2} d \mathcal{H}^{n-1} d a \\
& \geq \operatorname{dist}^{2}(\tilde{K}, 0) \int_{0}^{\epsilon} \mathcal{H}^{n-1}\left(\left\{d_{\partial \Omega}=a\right\}\right) d a \\
& \geq C \operatorname{dist}^{2}(K, M) \epsilon .
\end{aligned}
$$

This concludes the proof of the lower bound.

The proof of Propositions 2.3 and 2.5 are analogous, so we only point out the main changes.

Proof of Proposition 2.3 and 2.5. The argument for Proposition 2.3 follows along the same lines as the argument for Theorem 2. Indeed, passing from $u$ to $v(x)=$ $u(x)-M x-b$, it suffices to prove

$$
\tilde{E}_{\epsilon}(v):=\int_{\Omega} \operatorname{dist}^{2}(\nabla v, \tilde{K})+\epsilon\left|\nabla^{2} v\right|\left(\mathbb{R}^{n}\right) \geq C_{\Omega, K} \min \left\{\operatorname{dist}(M, K), \operatorname{dist}^{2}(M, K)\right\} \epsilon,
$$

where $\tilde{K}=K-M$. Arguing as in (59), the co-area formula implies

$$
\int_{\mathbb{R}^{n}} \operatorname{dist}^{2}(\nabla v, \tilde{K}) d x=\int_{0}^{\infty} \int_{\left\{d_{\partial \Omega}(x)=a\right\}} \operatorname{dist}^{2}(\nabla v, \tilde{K}) d \mathcal{H}^{n-1} d a .
$$

Next, for functions with $\nabla v \in C_{0}^{1}(\Omega)$ the fundamental theorem entails that for every $x \in \Omega$ and $y \in \mathbb{R}^{n} \backslash \bar{\Omega}$

$$
|\nabla v(x)| \leq \int_{0}^{1}\left|\nabla^{2} v(t x+(1-t) y)\right| d t|x-y|
$$

Hence, by approximation, integration in $\left\{x \in \Omega: \frac{\epsilon}{2} \leq d_{\partial \Omega}(s) \leq \epsilon\right\}$ for $\epsilon>0$ small, and division by $\epsilon>0$, for functions with $\nabla v \in W_{0}^{1,1}(\Omega)$ we obtain that

$$
\int_{\mathbb{R}^{n}}\left|\nabla^{2} v\right| d x \geq C_{\Omega} \int_{\left\{\frac{\epsilon}{2} \leq d_{\partial \Omega} \leq \epsilon\right\}} \frac{|\nabla v|}{d_{\partial \Omega}} d x=C_{\Omega} \int_{\frac{\epsilon}{2}}^{\epsilon} \int_{\left\{d_{\partial \Omega}=a\right\}} \frac{|\nabla v|}{a} d \mathcal{H}^{n-1} d a .
$$

In order to exploit this, we approximate $v$ by $v_{\nu}=v * \varphi_{\nu}$, where $\varphi_{\nu}$ is a standard mollifier. Using the notation $\Omega_{\nu}:=\left\{x \in \mathbb{R}^{n}: \operatorname{dist}(x, \Omega) \leq \nu\right\}$ for the extension $\nu$ of $\Omega$ and observing that $\operatorname{supp}\left(v_{\nu}\right) \subset \Omega_{\nu}$, we obtain

$$
\begin{aligned}
\int_{\mathbb{R}^{n}}\left|\nabla^{2} v_{\nu}\right| d x & \geq C_{\Omega} \int_{\left\{\frac{\epsilon}{2} \leq d_{\partial \Omega_{\nu}} \leq \epsilon\right\}} \frac{\left|\nabla v_{\nu}\right|}{d_{\partial \Omega_{\nu}}} d x \\
& \geq C_{\Omega} \int_{\frac{\epsilon}{2} \int_{\left\{d_{\partial \Omega_{\nu}}=a\right\}} \frac{\left|\nabla v_{\nu}\right|}{a} d \mathcal{H}^{n-1} d a .}
\end{aligned}
$$


Now, on the one hand, we have that $\int_{\mathbb{R}^{n}}\left|\nabla^{2} v_{\nu}\right| d x \leq\left|\nabla^{2} v\right|\left(\mathbb{R}^{n}\right)$. On the other hand, we have that $\nabla v_{\nu} \rightarrow \nabla v$ strongly in $L^{1}\left(\mathbb{R}^{n}\right)$ and as pointwise limits

$$
d_{\partial \Omega_{\nu}} \rightarrow d_{\partial \Omega}, \chi_{\Omega_{\nu}} \rightarrow \chi_{\Omega}
$$

where for an open set $U$ the function $\chi_{U}$ denotes the associated characteristic function. For the distance functions, the convergence follows from the regularity of $\Omega$ (which can for instance be seen in boundary geodesic coordinates). Hence, by dominated convergence and using that we infer from (63) that

$$
\left|\nabla^{2} v\right|\left(\mathbb{R}^{n}\right) \geq C_{\Omega} \int_{\frac{\epsilon}{2}}^{\epsilon} \int_{\left\{d_{\partial \Omega}=a\right\}} \frac{|\nabla v|}{a} d \mathcal{H}^{n-1} d a .
$$

Combining (62) and (64) as above then yields

$$
\tilde{E}_{\epsilon}(v) \geq C_{\Omega} \int_{\frac{\epsilon}{2}}^{\epsilon} \int_{\left\{d_{\partial \Omega}=a\right\}} \operatorname{dist}^{2}(\nabla v, \tilde{K})+|\nabla v| d \mathcal{H}^{n-1} d a .
$$

As $\tilde{K} \subset \mathbb{R}^{n \times n}$ is bounded and as

$$
\operatorname{dist}^{2}(\nabla v, \tilde{K})+|\nabla v| \geq\left\{\begin{array}{l}
\operatorname{dist}(\nabla v, \tilde{K})+|\nabla v| \geq \operatorname{dist}(M, K) \text { if } \operatorname{dist}(\nabla v, \tilde{K}) \geq 1 \\
\operatorname{dist}^{2}(\nabla v, \tilde{K})+C_{\tilde{K}}|\nabla v|^{2} \geq c_{\tilde{K}} \operatorname{dist}^{2}(M, K) \text { else }
\end{array}\right.
$$

where $C_{\tilde{K}}=\frac{1}{\sup \{\|A\|: A \in \tilde{K}\}}$. This implies

$$
\begin{aligned}
\tilde{E}_{\epsilon}(v) & \geq C_{\Omega} \int_{\frac{\epsilon}{2}}^{\epsilon} \int_{\left\{d_{\partial \Omega}=a\right\}} \min \left\{\operatorname{dist}(M, K), \operatorname{dist}^{2}(M, K)\right\} d \mathcal{H}^{n-1} d a \\
& \geq C_{K, \Omega} \epsilon \min \left\{\operatorname{dist}(M, K), \operatorname{dist}^{2}(M, K)\right\} .
\end{aligned}
$$

Similarly, we observe that for any $p \in(1, \infty)$

$$
\left\|\nabla^{2} v\right\|_{L^{p}(\Omega)}^{p} \geq C_{\Omega} \int_{\frac{\epsilon}{2}}^{2 \epsilon} \int_{\left\{d_{\partial \Omega}=a\right\}} \frac{|\nabla v|^{p}}{a^{p}} d \mathcal{H}^{n-1} d a .
$$

Combining this with (62) and the observation that for any $p \in(1,2]$, we can estimate

$$
\operatorname{dist}^{2}(\nabla v, \tilde{K})+|\nabla v|^{p} \geq C_{\tilde{K}, p} \min \left(\operatorname{dist}(M, K)^{2-p}, \operatorname{dist}^{2}(M, K)\right),
$$

where we used that $p \leq 2$ and thus

$$
|\nabla v|^{p} \geq \frac{|\nabla v|^{2}}{(\sup \{\|A\|: A \in \tilde{K}\})^{2-p}},
$$

also implies the lower bound for $p \in(1,2)$. This concludes the proof of Propositions 2.3 and 2.5 .

\section{Flexibility}

In this section, we recall the convex integration results from [RZZ16, RZZ17] in the model case of the geometrically linearised hexagonal-to-rhombic phase transformation (which is discussed in Section 3.1) and present implementations of these convex integration schemes.

We hope that eventually these numerical implementations will allow us to compare experimental results with our theoretical findings, thus leading to an improved understanding of whether convex integration solutions are really physical or purely mathematical artefacts. We begin this discussion by briefly recalling 


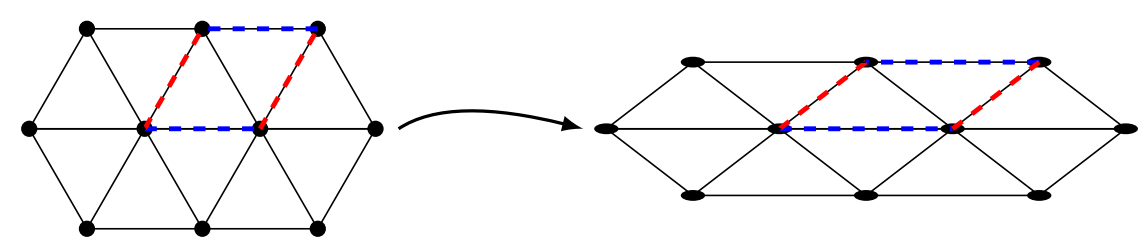

FiguRE 1. A microscopic derivation of the hexagonal-to-rhombic phase transformation: A hexagonal lattice (which can also be interpreted to consist of a rhombic lattice) is deformed by stretching/compressing the sides of the rhombus. Carrying this out while preserving the volume and linearising the resulting deformation gradients leads to the (infinitesimal) transformation strains $e^{(1)}, e^{(2)}, e^{(3)}$.

the two-dimensional, geometrically linearised hexagonal-to-rhombic phase transformation in Section 3.1 and the properties of the convex integration algorithms from [RZZ16, RZZ17] in Section 3.2. In Section 3.3 we will then discuss (nonquantitative) numerical implementations of the schemes and compare them. In Section 3.4 we address more quantitative properties of our numerical implementations by numerically analysing the involved emerging length scales (which can be viewed as a measure of the complexity of the solutions).

3.1. The geometrically linearised hexagonal-to-rhombic phase transformation. We recall the model and some properties of the geometrically linearised hexagonal-to-rhombic phase transformation.

A difficulty of the variational model from Section 1.1 is the two-fold "nonlinearity" of the problem, manifested in the two physical requirements (i), (ii). This is also still reflected in the $m$-well problem from Section 1.2, in that the set $K(\theta)$ still displays $S O(3)$ invariance, and has a multi-well structure at temperatures below $\theta_{c}$. Hence, in order to simplify this, it is often convenient to "linearise" the frame indifference assumption and to pass from $S O(3)$ to $S k e w(3)$ invariance (we recall that $\operatorname{Skew}(3)$ is the linearisation of $S O(3)$ at the identity). Mathematically, this has the advantage of dealing with an invariance which is given by a vector space structure in contrast to a nonlinear group structure, while at the same time preserving the "material nonlinearity", i.e. the multi-well structure. For one-well problems this has been rigorously justified in [DMNP02, ABK15]. In the small rotation regime, it is expected that this linearisation still captures important features of the nonlinear original problem (3) (although care is required, in particular in the large rotation regime, see [Bha93]).

In the sequel, we study such a geometrically linearised problem in two-dimensions. Passing formally from the deformation $y(x)$ to the displacement $u(x)=y(x)-x$ and dropping all "higher order terms" (see the discussion in [Bha03, Chapter 11]), this turns the nonlinear problem (3) (at fixed temperature $\theta<\theta_{c}$ ) into a linearised $m$-well problem of the type

$$
e(\nabla u):=\frac{1}{2}\left(\nabla u+(\nabla u)^{t}\right) \in\left\{e^{(1)}, \ldots, e^{(m)}\right\}+\operatorname{Skew}(3) .
$$

Here $e(\nabla u)$ denotes the (infinitesimal) strain tensor and $e^{(1)}, \ldots, e^{(m)} \in \mathbb{R}_{\text {sym }}^{3 \times 3}$ represent the variants of martensite. We emphasise that although we have linearised the geometric nonlinearity of the problem by passing from an $S O(3)$ invariance to a Skew(3) invariance, we have preserved the main material nonlinearity in the model, i.e. the presence of multiple wells. 
As a next step, we specify the transformation to be the two-dimensional hexagonal-to-rhombic phase transformation (which is related to transformations occurring in materials such as $\mathrm{Mg}_{2} \mathrm{Al}_{4} \mathrm{Si}_{18}, \mathrm{Mg}$-Cd alloys or $\mathrm{Pb}_{3}\left(\mathrm{VO}_{4}\right)_{2}$, see [KK91, CPL14]), see also Figure 1 for a microscopic two-dimensional derivation of the deformation matrices (the third direction can be ignored as the material only undergoes an affine change there). Assuming that the material undergoes at most an affine deformation in the third direction, allows us to reduce the three-dimensional problem to a two-dimensional one. Hence, in the following we study the differential inclusion

$$
e(\nabla u):=\frac{1}{2}\left(\nabla u+(\nabla u)^{t}\right) \in\left\{e^{(1)}, e^{(2)}, e^{(3)}\right\}+\operatorname{Skew}(2),
$$

where now $u: \Omega \subset \mathbb{R}^{2} \rightarrow \mathbb{R}^{2}$ and

$$
e^{(1)}=\left(\begin{array}{cc}
1 & 0 \\
0 & -1
\end{array}\right), e^{(2)}=\frac{1}{2}\left(\begin{array}{cc}
-1 & \sqrt{3} \\
\sqrt{3} & 1
\end{array}\right), e^{(3)}=\frac{1}{2}\left(\begin{array}{cc}
-1 & -\sqrt{3} \\
-\sqrt{3} & 1
\end{array}\right) .
$$

Note that the traces of all three matrices in (68) vanish, which corresponds to the modelling assumption that the transformation is (infinitesimally) volume preserving.

Important features of this model which make it a good candidate to study flexibility properties of phase transformations in solids are:

- All three matrices in (68) are symmetrised rank-one connected. Hence, it is possible to form twins between all of these.

- We have $K^{l c}=\operatorname{conv}\left(e^{(1)}, e^{(2)}, e^{(3)}\right)$. Hence, after pulling up the problem into full matrix space (by adding the skew direction) we are dealing with a co-dimension one problem in which the only constraint is given by the vanishing trace condition.

- There are interesting experimental and numerical results on this phase transformation, see [KK91, CPL14].

Let us finally comment on our choice of dealing with a geometrically linearised problem. From the mathematical point of view, it has the advantage that it allows us to concentrate on the analytic aspects of convex integration, ignoring the possibly non-trivial geometry in matrix space. Comparing the predicted microstructures which are obtained within the linearised theory (see [CPL14] and Section 7 in [RZZ16]) with experiments on such materials [KK91] indicates that many important and interesting microstructures (including the star-shaped ones which are important in the context of convex integration) match very well. Moreover, in both of our convex integration algorithms [RZZ16, RZZ17] it was possible to control the size of the skew matrices that are needed in the constructions (to be bounded by a fixed constant of the order one). Hence, although being aware of the fact that the linearised theory of elasticity is always an approximation with limitations to its validity, we believe that in our situation the analysis of geometrically linearised phase transformations provides an interesting and legitimate first step towards a better understanding of convex integration solutions.

Due to the large lamination convex hull of the hexagonal-to-rhombic phase transformation, it is possible to construct a plethora of convex integration solutions:

Theorem 3 (Existence of convex integration solutions). Let $\Omega \subset \mathbb{R}^{2}$ be an open Lipschitz domain. Let $e^{(1)}, e^{(2)}, e^{(3)}$ be as in (68). Then for any $M \in \mathbb{R}^{2 \times 2}$ with

$$
e(M):=\frac{1}{2}\left(M+M^{t}\right) \in \operatorname{intconv}\left(\left\{e^{(1)}, e^{(2)}, e^{(3)}\right\}\right)
$$

there exists a solution $u$ of (67). Moreover, it is possible to ensure that for any $\epsilon>0$ there is a solution $u_{\epsilon}$ to (67) with

$$
\left\|u_{\epsilon}-M x\right\|_{L^{\infty}(\Omega)}<\epsilon .
$$



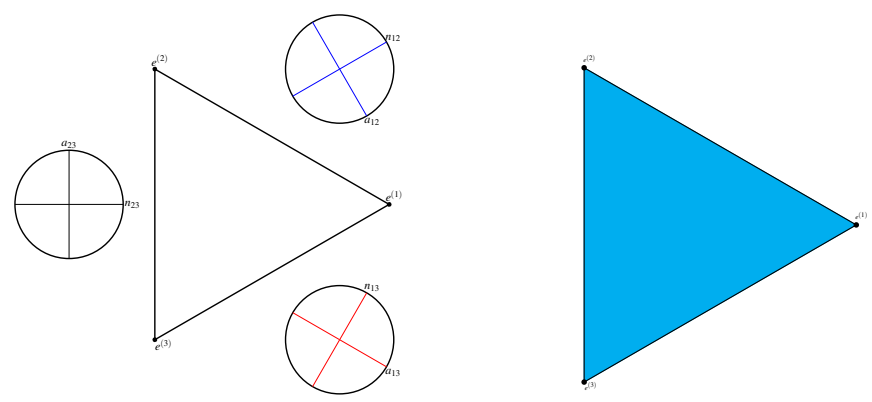

FiguRE 2. Left panel: The symmetrised rank-one connections between the wells: The triangle depicts the equilateral triangle that is spanned in strain space by the wells $e^{(1)}, e^{(2)}, e^{(3)}$. These are pairwise symmetrised rank-one connected with two possible rank-one connections each (due to the trace free constraint these are related by $\frac{\pi}{2}$-rotations). The orientation of these rank-one directions are depicted in the circles which are drawn next to the corresponding sides of the equilateral triangle. Here the choice of the sign of the normals is still free, depending on the ordering of the wells.

Right panel: The laminar convex hull of the wells $e^{(1)}, e^{(2)}, e^{(3)}$. Using two-fold laminations, it is possible to reach any boundary datum in the interior of the convex hull of the three wells $e^{(1)}, e^{(2)}, e^{(3)}$.

This (non-quantitative) result follows for instance from the arguments of MüllerŠverák [MŠ99] or from the results of Dacorogna-Marcellini [DM95b]. As seen from the approximation result (69) these solutions are highly non-unique; in [DM95b] this is made more precise in a Baire category sense. We remark that in invoking the arguments from [MŠ99] and [DM95b], there is a slight subtlety here, in that the inclusion is formulated on the level of the symmetrised gradient and not on the level of the full gradient. However, there are various ways of overcoming this, one being to simply "pull up" the inclusion problem to an inclusion problem for the full gradient.

In [RZZ16] and [RZZ17] together with B. Zwicknagl we analysed the underlying construction schemes more precisely and showed that it is possible to improve the regularity of solutions to $(67)$ on a $\left(W^{s, p}\right)$ Sobolev scale by choosing the underlying lengths scales carefully. The iterative algorithms in [RZZ16] and [RZZ17] however differed quantitatively, in that they produced different dependences: While the regularity of solutions constructed by the scheme from [RZZ16] depended on the position of the boundary data $M$ in matrix space, the scheme from [RZZ17] could produce solutions with a regularity which did not depend on this (or more precisely, where we could give bounds which were independent of this).

In the sequel, we discuss and compare explicit numerical implementations of these schemes, which illustrate the differences which occur here. While convex integration solutions in the context of elasticity have been known for quite a while, the present note seems to contain the first numerical implementation of these. In our numerics, we focus on the qualitative convex integration result from Theorem 3 and do not seek to optimize the underlying partitions, which was necessary in the quantitative analysis in [RZZ16, RZZ17]. This is due to the introduction of highly fractal structures which would exceed the capability of our computers (and which from a certain level onwards would also not be seen as major changes without zooming into the structures). Also, we believe that, while being analytically convenient, 
the exact coverings from [RZZ16, RZZ17] are far from optimal. Since the numerical implementation without this already provides interesting insights, we opted to focus on the problems without the additional layer of (analytical and numerical) difficulty originating from the complicated covering structures from [RZZ16, RZZ17].

3.2. The outline of the convex integration scheme for the hexagonal-torhombic phase transformation. We briefly describe the main scheme of our convex integration algorithms. They are iterative constructions in which a given gradient distribution is iteratively replaced by a distribution whose symmetrised gradients approach the wells. Schematically, the algorithms carry out the following steps:

(i) On a given piecewise polygonal (or Lipschitz) domain, we start with a constant deformation gradient $M$ with $e(M) \in \operatorname{int} \operatorname{conv}\left\{e^{(1)}, e^{(2)}, e^{(3)}\right\}$.

(ii) In a suitable model domain, we apply a replacement construction which allows us to replace the given gradient by a new distribution of finitely many gradients whose level sets are polygonal in such a way that

(a) the new gradient distribution attains values only in (intconv $\left\{e^{(1)}, e^{(2)}, e^{(3)}\right\}+$ Skew $(2)) \cup(\hat{K}+\operatorname{Skew}(2))$, where $\hat{K}=\left\{e^{(1)}, e^{(2)}, e^{(3)}\right\}$,

(b) the boundary conditions on the boundary of the model domain are not changed,

(c) the $L^{1}$ norm of the distance of the new symmetrised gradients to the wells $\hat{K}$ decreases by an exponential factor compared to the $L^{1}$ norm of $\operatorname{dist}(M, \hat{K})$.

(iii) We cover our domain or a sufficiently large volume fraction of our domain by the model domain and in each of the model domains we apply the replacement construction from step (ii).

(iv) We iterate this procedure on each of the level sets of the new gradient distribution.

A more detailed pseudocode variant of the algorithm is provided in the appendix and can also be found in [RZZ16, RZZ17]. The algorithms from [RZZ16, RZZ17] differ mainly in the replacement constructions. The two choices of the replacement algorithms are illustrated and explained in Figures 3 and 4.

3.3. Numerical implementation of the convex integration schemes for the hexagonal-to-rhombic phase transformation. In this section, we present some of the output of our convex integration algorithms in the two variants from [RZZ16, RZZ17]. We hope that eventually this can be compared to experimental results. Here we use a colour coding based on the CMYK colour model, where the colours cyan, magenta and yellow (we do not use key) correspond to one of the wells respectively. More precisely, the well $e^{(1)}$ corresponds to cyan, the well $e^{(2)}$ to magenta, and $e^{(3)}$ to yellow. A matrix which is included in the interior of the convex hull is correspondingly depicted as a convex combination of these colourings (see Figure 3).

We remark that in commercial printing the CMYK colour model is used to blend colours as mixtures of the primary colours cyan, magenta, yellow (and black). In particular, for our purposes this is very convenient, as it allows us to work with "barycentric coordinates" based on cyan, magenta and yellow. Moreover, too fine structures in our construction, which are hard to see in the picture due to limited resolution and rasterisation, are then "homogenised" automatically in a way which agrees with the colours of the averaged matrix values.

We compare the output of the two convex integration algorithms from [RZZ16, RZZ17] for three different scenarios. These are chosen such that the overall boundary data $M \in \operatorname{intconv}\left(\left\{e^{(1)}, e^{(2)}, e^{(3)}\right\}\right)$ are such that either (see Figure 5) 

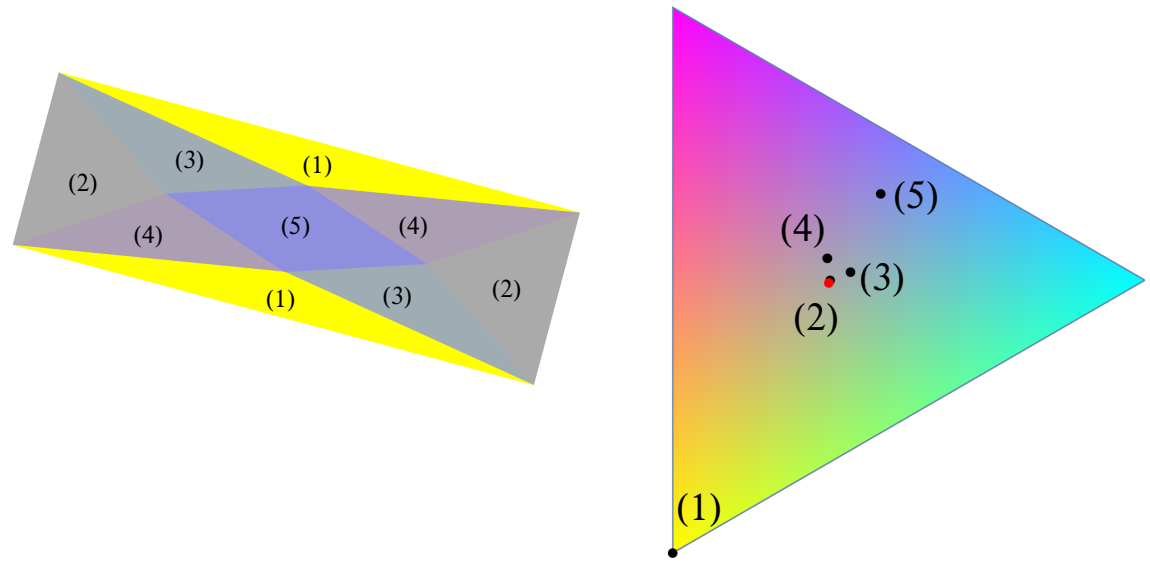

Figure 3. Left panel: The replacement construction from [RZZ16]. The triangles correspond to the level sets of the replacement function (with different colours which are encoded through the CYMK colour coding corresponding to different values of the symmetrised gradient as shown in the right panel). In the replacement construction from [RZZ16] on a fixed volume fraction of the domain (here labelled as (1)), the new gradient is exactly in the well (here this corresponds to the well $e^{(3)}$ illustrated in yellow). In the remainder of the domain (i.e. on the level sets which are labelled as (2)-(5)) the symmetrised gradients do not approach the wells, but are on the contrary even "pushed further into the interior" of intconv $\left(e^{(1)}, e^{(2)}, e^{(3)}\right)$ (for which we refer to the right panel and the associated deformation matrices which are also marked as (2)-(5)).

Right panel: The replacement construction from [RZZ16] in symmetric, trace-free matrix space depicted in CMYK colour encoding (where we do not use key). The colour triangle corresponds to the convex hull of the wells, in that $e^{(1)}$ is depicted as cyan, $e^{(2)}$ as magenta and $e^{(3)}$ as yellow. Correspondingly convex combinations are presented as blends of these colours. The algorithm from [RZZ16] starts with a given boundary datum (here marked as the red point which is roughly in the center of the equilateral triangle) and replaces it by five new matrices (marked in black and labelled as (1)-(5) as in the left panel), of which one is exactly in the wells (here the yellow well, labelled as (1)), and four in the interior. In particular, we note that some of the new data are "pushed" further into the interior of the convex hull of $e^{(1)}, e^{(2)}, e^{(3)}$. On this part of the domain the gradient distribution does not improve at all.

(i) $M$ is close to the barycenter $\frac{1}{3} e^{(1)}+\frac{1}{3} e^{(2)}+\frac{1}{3} e^{(3)}=0$ of the equilateral triangle spanned by $e^{(1)}, e^{(2)}, e^{(3)}$.

(ii) $M$ is close to one of the wells.

(iii) $M$ is close to the boundary of $\operatorname{intconv}\left(e^{(1)}, e^{(2)}, e^{(3)}\right)$ and essentially in between two wells.

We remark that, due to the $S k e w(2)$ symmetry, our convex integration construction essentially only depends on the symmetric part $e(M)$ of the matrix $M$ (the skew 

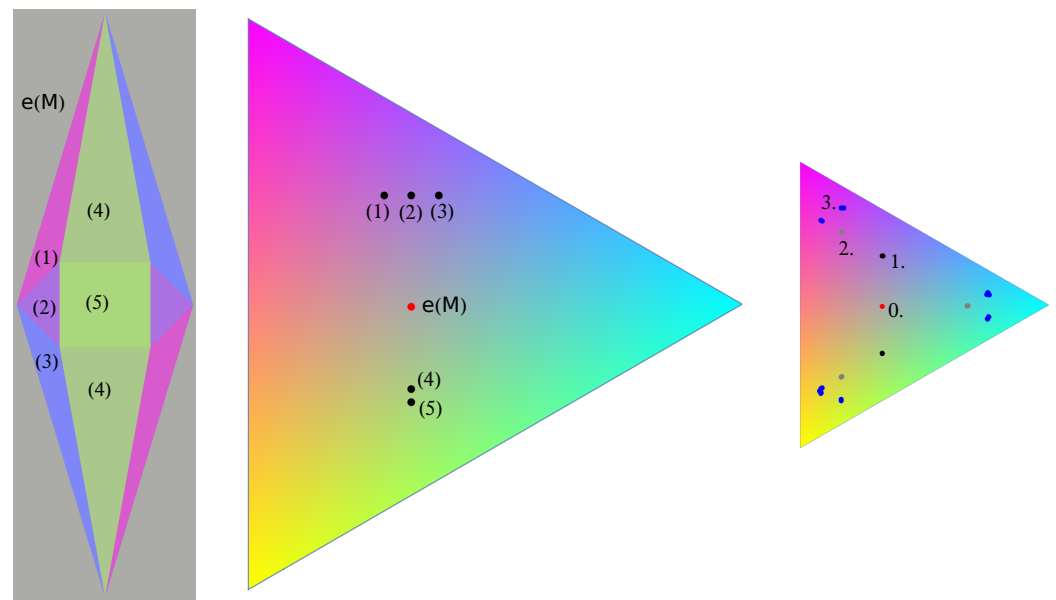

FiguRE 4. The replacement construction from [RZZ17].

Left Panel: The gradient distribution is modified in a diamond-shaped domain. The boundary datum is a linear map whose deformation gradient is close to the barycenter of the set intconv $\left\{e^{(1)}, e^{(2)}, e^{(3)}\right\}$. In the center panel, it corresponds to the red dot labelled as $e(M)$ (which in the CYMK colour coding is grey). It is thus encoded in grey on the outside of the diamond in the left panel. Again the triangles within the diamond correspond to the level sets of the replacement function with matching labels on the left and center picture. The different colours correspond to different values of the symmetrised gradient, numbered (1)-(5) and as shown in the center panel.

Center panel: As in Figure 3, in the center we have plotted the position of the new distribution of the symmetrised gradients in intconv $\left\{e^{(1)}, e^{(2)}, e^{(3)}\right\}$ obtained after applying the replacement construction from [RZZ17] starting with a gradient whose symmetric gradient is $e(M)$ (i.e. the red dot in the online version). The replacement construction from [RZZ17] is a perturbation of a laminate. In a first iteration of the construction, the red dot is replaced by five black dots. For illustration purposes, here and on the left we show the picture for comparatively large error tolerance in the convex integration scheme, since otherwise the respective points (1)-(3) and (4),(5) and the corresponding colors would be visually indistinguishable.

Right panel: The construction shown on the left and center thus allows us to replace a single matrix, marked as the 0th step, by five new matrices, marked as the 1st step, within a rectangular domain. We note here that the matrices are shown with our actual choice of error tolerance, which is smaller than on the left and center. Hence several of these five points visually overlap. Iterating the convex integration algorithm, each of the five matrices will then in turn be replaced by five new matrices, marked as the 2nd step, and iterating further by again five times as many matrices, marked as the 3rd step. We note the "uniformity" of the algorithm, which is reflected in the fact that the successive iterations approach the wells uniformly (in barycentric coordinates).

part only amounts to orthogonal translations of the matrices). We discuss the model cases (i)-(iii) separately in the sequel.

3.3.1. An example of case (i). As a first case, we discuss boundary data which are chosen to lie very close to the barycenter of the equilateral triangle spanned by the matrices $e^{(1)}, e^{(2)}, e^{(3)}$.

In the implementations of the two algorithms from [RZZ16] and [RZZ17] we see major differences, which are illustrated in Figure 7. We observe the following differences:

- finiteness: The construction of [RZZ16] is "finite" in the sense that for almost all points $x \in \Omega$ there exists an iteration step $k$ (which depends on 


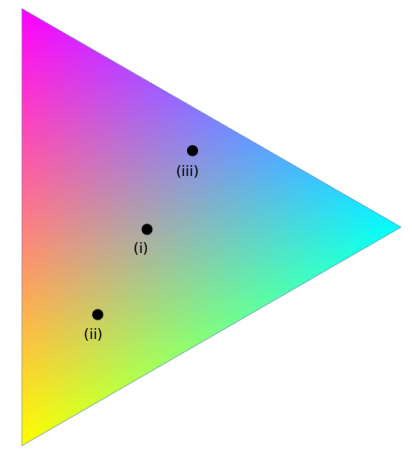

FiguRE 5. The three cases (i)-(iii). The matrices marked in the triangle show the location of the boundary data from cases (i)-(iii) in the equilateral triangle spanned by $e^{(1)}, e^{(2)}, e^{(3)}$.

$x$ ) such that after $k$ steps the gradient distribution at $x$ is already in the wells $\hat{K}$. In our example this is manifested in the fact that already after three iteration steps there are large patches (approximately 20 percent), in which the final colouring is attained (in Figure 7 for instance there are large yellow patches). In contrast to this, the algorithm from [RZZ17] is "countably infinite", i.e. in general there are no points in which the gradient is already exactly in the well after a finite number of iteration steps: Although in the fine microstructure one already sees colours close to the wells, these are not exactly in the wells in general. In particular this yields a very fine-scale microstructure, which "homogenises" in the "eye norm".

- uniformity: In the implementation of the algorithm [RZZ16] the replacement constructions always contain patches which are "pushed away" into the interior (see Figure 3). In the implementation of the algorithm from [RZZ17], we in contrast see that the replacement constructions use constructions whose colours are increasingly close to the wells (see Figure 4, right panel).

- normal directions: While the normals which are used in the algorithm from [RZZ16] may vary in a continuum, the ones in the algorithm from [RZZ17] are discrete (they correspond to the directions of the symmetrised rank-one directions of the wells). Although this is hard to see by the "eye norm", it is manifested in the replacement constructions shown in Figure 8.

Both constructions are of self-similar, fractal structure (however leading to quite different overall structures).

3.3.2. An example of case (ii). In addition to the differences which had already been discussed in the case (i), the most striking point which can is reflected in the implementations of the case (ii) is the "inertia" which is underlying the algorithm from [RZZ16] before the well is changed. This is a consequence of the choice of the replacement algorithm from [RZZ16]: Here the original boundary data are replaced by five new matrices, of which some are "pushed further into the interior" of the convex hull of the strains $e^{(1)}, e^{(2)}, e^{(3)}$ (see Figure 3). This implies that the closest well eventually changes. However, since the push-out is achieved with a small fixed factor, this is a slower process, the closer the original boundary datum had been to one of the wells. In particular, if one starts with a matrix very close to one of the wells, it might take a large number of iteration steps before any other well is used 


\begin{tabular}{l|llll} 
& $\begin{array}{l}\text { Number of itera- Local convergence } \\
\text { tions until } \hat{K} \text { is } \\
\text { reached }\end{array}$ & $\begin{array}{l}\text { Number of direc- } \\
\text { tions }\end{array}$ \\
\hline [RZZ16] & $\begin{array}{l}\text { finite depending on } \\
\text { point }\end{array}$ & $\begin{array}{l}\text { exactly attained, continum of direc- } \\
\text { after non-uniform tions in cones } \\
\text { number of steps; } \\
\text { partially pushed }\end{array}$ \\
[RZZ17] & countably infinite the interior & $\begin{array}{l}\text { uniform, exponen- finitely many direc- } \\
\text { tial convergence tions } \\
\end{array}$ \\
& & rate & \\
\hline
\end{tabular}

FiguRE 6. A summary and comparison of the properties of the two replacement functions described above.

(as the algorithm will have reached very small scales by then, it is also hard to see this, even if this happens).

In contrast to this, the algorithm from [RZZ17] uses values close to two of the wells in each iteration step: Hence even if one starts close to a single well, the other wells will emerge within the next two iteration steps (see the second panel of Figure 11).

Again both of the constructions (the one from [RZZ16] and the one from [RZZ17]) already display fractal behaviour.

3.3.3. An example of case (iii). Last but not least, we discuss the setting in which the boundary data are very close to the rank-one line between two wells. Here both constructions mainly consist of shades of cyan and magenta. The angle between the closest well and the boundary is very flat. Hence, we expect that the construction from [RZZ16] (left panel in Figure 10) is forced to use very fine scales depending on the boundary distance. In particular, the regularity in the finely twinned areas should be bad, eventually yielding regularities which are significantly rougher than the ones from cases (i), (ii) (see Figures 7, 9). In contrast, the construction from [RZZ17] (right panel in Figure 10) is not significantly more complex than the other constructions from Figures 11. 

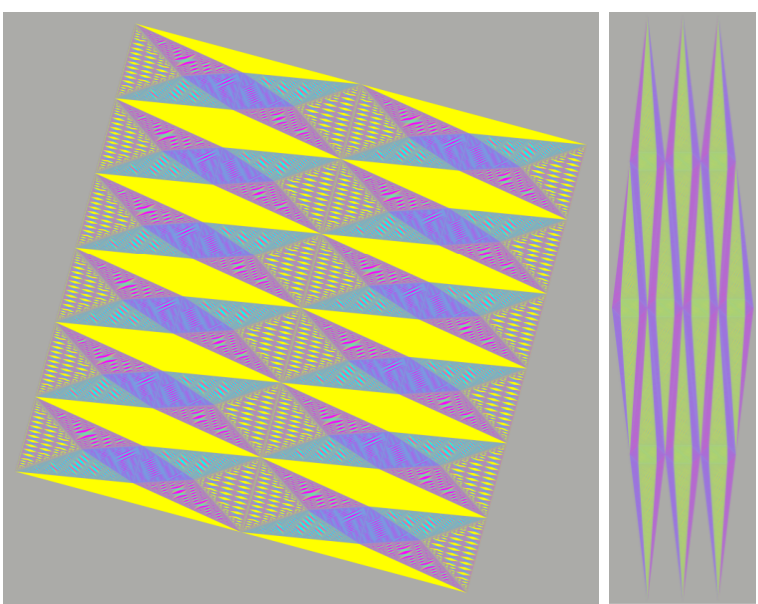

FiguRE 7. Comparison of the algorithms from [RZZ16, RZZ17] in the case (i):

Both pictures illustrate the solution of our convex integration algorithms with the choice of boundary data of the form $M=0.33 e^{(1)}+0.33 e^{(2)}+0.34 e^{(3)}$, which is represented as a grey background color as in Figure 5 .

Left panel: The left panel illustrates the output of the algorithm from [RZZ16], which we apply in the interior square domain $\Omega$ with the prescribed boundary data. Here within the square the largest patches correspond to the majority phase, i.e. yellow. Although we have only ploted three iterations of the algorithm (finer scales cannot be distinguished by the eye), we can already clearly see the emergence of the three wells in large yellow, cyan and magenta patches. We also see the fractal structure of the solutions.

Right panel: Compared to the algorithm from [RZZ16], the diamond construction from [RZZ17] displays a more "fine scale" structure (we refer to Figure 11 for a detailed view) and here is applied on a different domain consisting of a union of diamonds (as these are the building block constructions of the convex integration algorithm from [RZZ17]). Again we have depicted the result of the convex integration algorithm after three iteration steps and have used the same boundary data as in the left panel. Since however these boundary values are close to the barycenter of the equilateral triangle spanned by $e^{(1)}, e^{(2)}, e^{(3)}$, the diamond construction does not yet display the effects of the strong geometric convergence, which it has for data closer to the wells (these effects would become apparent after a few more iterations). We remark that in this second construction no purely cyan, magenta or yellow patches are present yet, but that colors only successively get closer to these (compare Figure 4, right panel). 

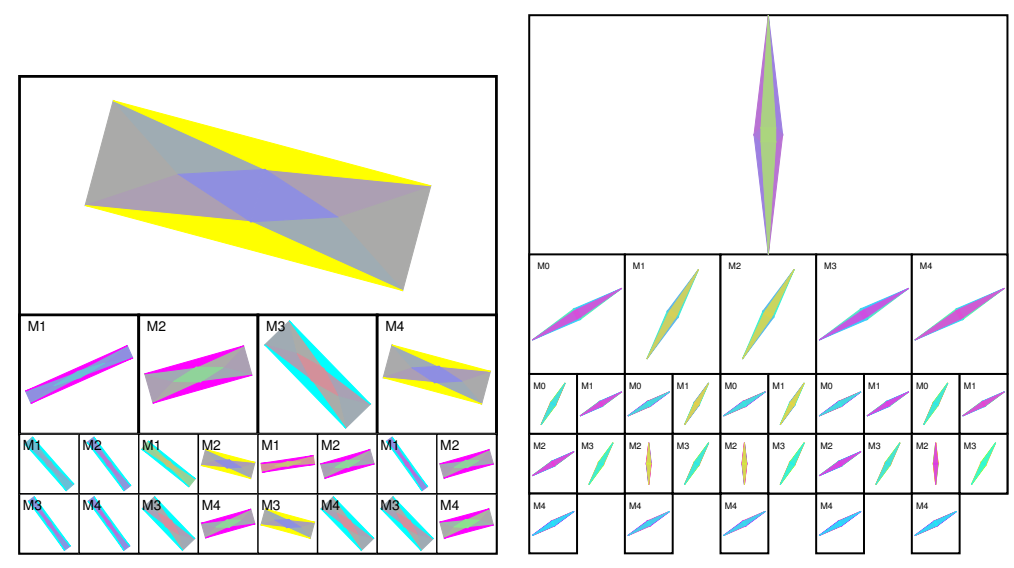

FiguRE 8. The replacement constructions which are used in the algorithm leading to the constructions in Figure 7.

Left panel: The rectangle on top depicts the replacement construction as shown in Figure 3, where the matrix $M=0.33 e^{(1)}+0.33 e^{(2)}+0.34 e^{(3)}$ is replaced by a matrix in the well, which is colored yellow and unchanged in further iterations, and four other matrices $M_{1}, \ldots, M_{4}$. Each of these four matrices $M_{1}, \ldots, M_{4}$ is then replaced using another application of the construction, which is depicted in the four medium-sized rectangles below it. In turn the four small rectangles below each replacement depict the next iteration of the replacement construction.

Right panel: Here we apply the algorithm from [RZZ17] depicted in Figure 4 instead. The matrix $M=0.33 e^{(1)}+0.33 e^{(2)}+0.34 e^{(3)}$ is replaced by five matrices $M_{0}, \ldots, M_{4}$, of which none is exactly in a well. Hence, as opposed to the left panel, the iteration is repeated for all five matrices. Again, the figure should be read as a "tree" from top to bottom, where each row below another row replaces the corresponding construction.

Although it is not always easy to detect this by the "eye norm", the constructions which are used in the left panel are slightly rotated with respect to each other (e.g. the different replacement constructions involving yellow), while there are only a discrete number of orientations in the right panel. 


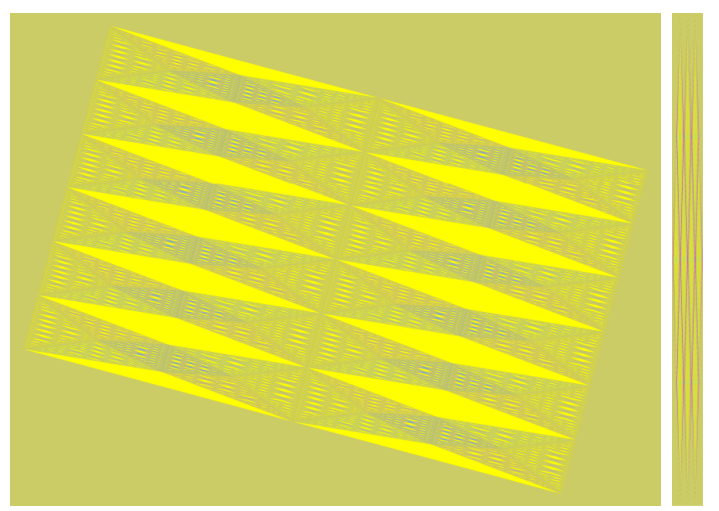

Figure 9. Comparison of the algorithms from [RZZ16, RZZ17] in the case (ii):

In this illustration of the algorithm we have chosen the boundary data to be $M=0.2 e^{(1)}+0.2 e^{(2)}+0.6 e^{(3)}$, which is shown as a dark yellow background color as in Figure 5. The construction on the left is shown for the domain given by the inner rectangle, while the one on the right is shown for a domain composed of a union of diamonds. General domains can then be approximated by tiling with rescaled copies of these constructions. One of the most striking differences after three iteration steps between the algorithms from [RZZ16] (left panel) and from [RZZ17] (right panel), is the fact that the first algorithm still essentially stays in a neighbourhood of a single well (only in the third iteration step a very small fraction of cyan emerges on small scales). In contrast, the algorithm from [RZZ17] always uses two wells in each iteration step. In particular, one clearly sees the emergence of magenta on the outer structures of the diamond and after zooming in also cyan can be recognized (see Figure 11, second panel, for a detailed view). 

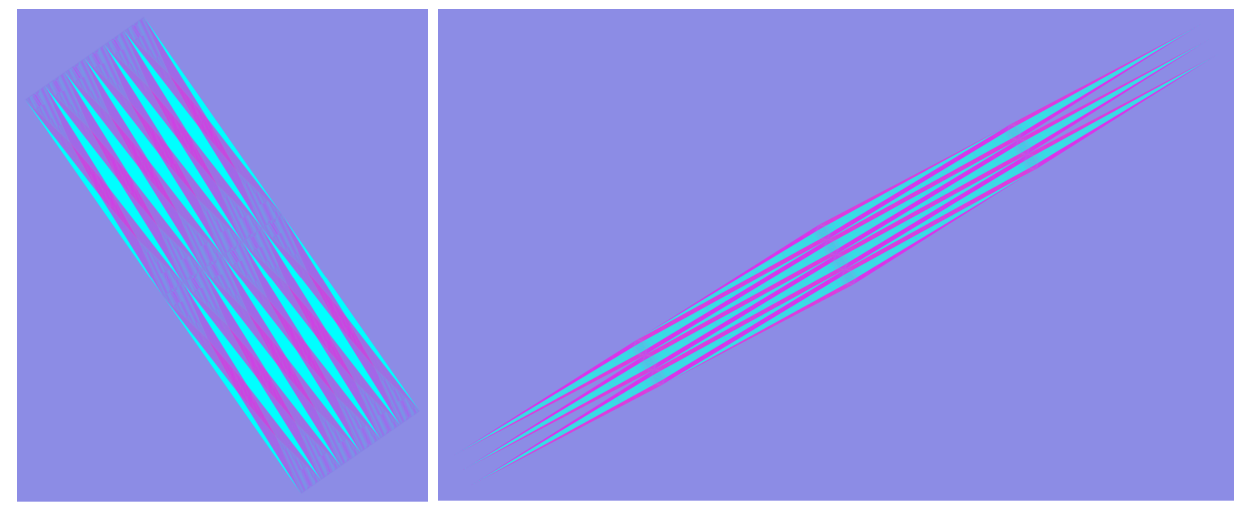

Figure 10. An implementation of the convex integration algorithm for boundary data which are very close to the boundary of the convex hull of the wells.

The boundary data are chosen to be $M=0.45 e^{(1)}+0.45 e^{(2)}+0.1 e^{(3)}$, which is shown as a purple background color as in case (iii) shown in Figure 5. The left panel shows the implementation of the algorithm from [RZZ16] on a domain given a rotated rectangle, the right panel the one from [RZZ17] on a domain given by a union of diamonds. The complexity of the right panel is comparable to the constructions from cases (i), (ii). Although this is not easy to detect by the eye norm, the complexity of the figure in the left panel is significantly more complex than the ones from these cases: The scales replacing the old structures are so fine, that they are homogenised by rasterisation in pictures of the algorithm and are hence hardly detectable (see Figure 11 for a detailed view). 

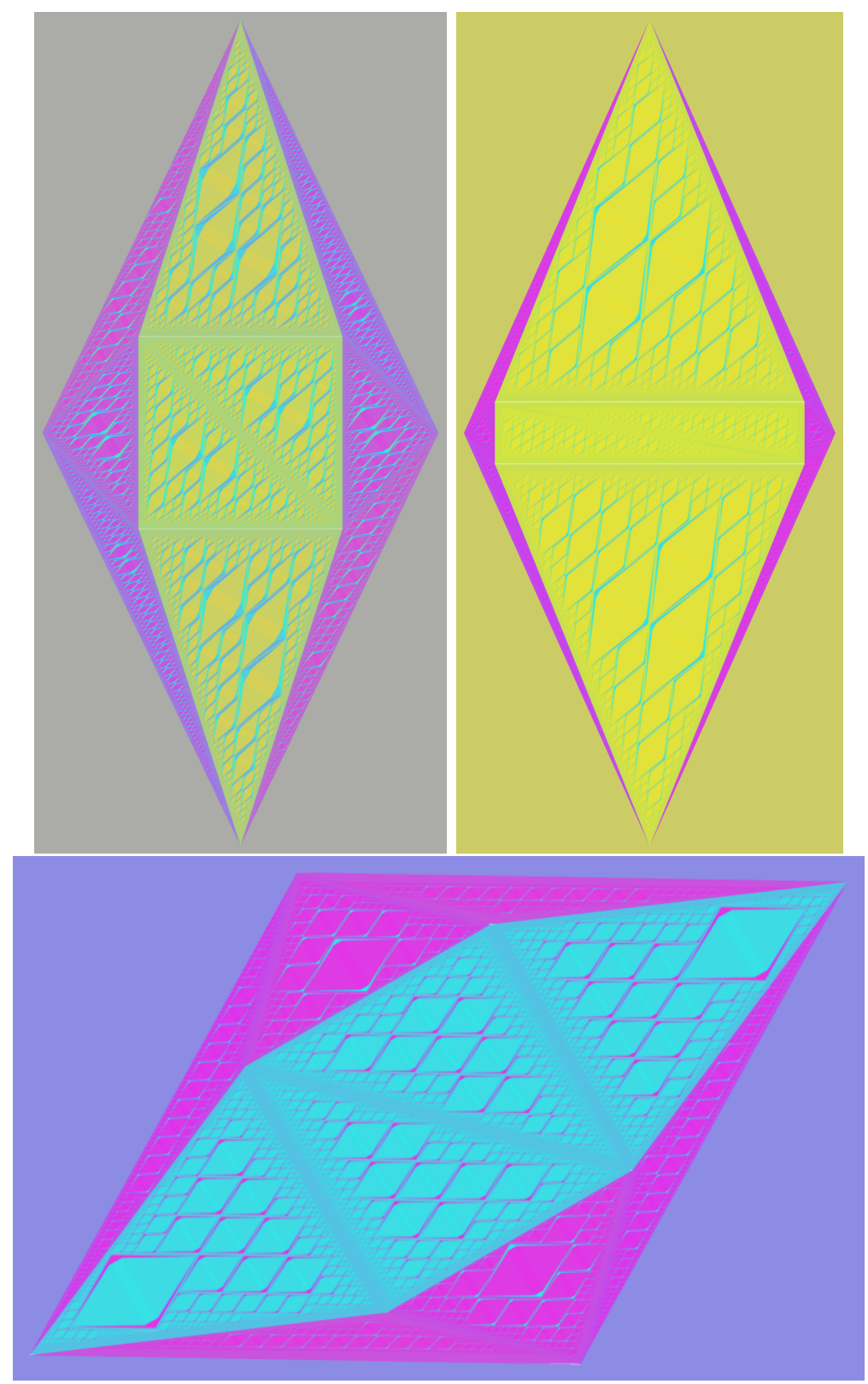

FigURE 11. The construction in [RZZ17] requires a small (but uniform) aspect ratio of the building block constructions in order to control the errors in matrix space. This results in very fine structures (see Figures 7, 9, 10, right panel). For illustration purposes, in the present figure we hence show the same constructions after three iteration steps but with an artificially increased aspect ratio (we have roughly increased the real aspect ratio by a factor 10) and for a domain given by a single diamond. From top to bottom and left to right this corresponds to the cases (i), (ii), (iii) discussed below and with corresponding background colors as in Figure 5 .

3.4. Numerical evaluation of the length scale and area distribution. In this section we augment our numerical implementations of the convex integration 
solutions from the previous section by more quantitative numerical results. These complement the regularity discussion from Section 2 by providing statistics on the occurring lengths and areas in the settings (i)-(iii) from Section 3.3. These data allow us to obtain numerical bounds on the regularity of the produced convex integration solutions. We refer to [PLKK97, BCH15, CH18, TIVP17] and the references therein where similar data were studied experimentally and theoretically for related models.

More precisely, we numerically implement the construction of the algorithm in [RZZ16] for seven iteration steps. Here, the length of the long side and the area of the rectangle construction depicted in Figure 3 provide natural scales. Due to computation times, we have limited our computation to length scales at least $10^{-4}$ and areas at least $10^{-9}$. Their histograms are shown using a log-log scale in Figures 13-15 for boundary data corresponding to the three cases of Figure 5. In particular, we observe that both quantities exhibit a power law behaviour:

$$
\begin{aligned}
\#\{\text { lengths at scale } x\} & \sim x^{-1-\sigma}, \\
\#\{\text { areas at scale } a\} & \sim a^{-1+\delta} .
\end{aligned}
$$

Since the underlying replacement construction does not further modify the region where $e(\nabla u)$ is in a well (region (1) in Figure 3), in the limit of no cut-off scale all of $\Omega$ is covered. Hence, given a cut-off length $\lambda$ and a corresponding area cutoff at $\lambda^{2}$, using the boundedness of the gradients in our construction, we may estimate the $L^{1}$ error (compared to the limit $\nabla u_{\infty}$ ) by

$$
\|\nabla u\|_{L^{1}} \leq C \int_{0}^{\lambda^{2}} a^{-1+\delta} d a=c_{\delta} \lambda^{2 \delta} .
$$

Conversely, the sum over all length scales provides a bound on the $B V$ norm by

$$
\|\nabla u\|_{B V} \leq C \int_{\lambda}^{1} x^{-1-\sigma} d x \approx c_{\sigma} \lambda^{-\sigma} .
$$

In our theoretic work from [RZZ16, RZZ17] we deduced higher Sobolev regularity estimates by interpolating the $B V$ and the $L^{p}$ (with $p \sim 1$ ) bounds for the deformation gradients. As our length and area distributions provide numerical bounds for these, we can use our data to infer explicit numerical values (in [RZZ16, RZZ17] we only considered the qualitative behaviour and did not try to optimise the quantitative bounds): By the interpolation results from [RZZ16] (Theorem 2) we have

$$
\|\nabla u\|_{W^{\theta, 1}} \leq C\|\nabla u\|_{B V}^{\theta}\|\nabla u\|_{L^{1}}^{1-\theta},
$$

where we have used the boundedness of our convex integration solutions. Denoting the exponents from the regressions in Figures 13 to 15 by $-1-\sigma_{j}$ (lengths) and $-1+\delta_{j}$ (areas) we have that for the deformations that are obtained at the end of our iteration

$$
\|\nabla u\|_{B V} \sim \lambda^{-\sigma_{j}},\|\nabla u\|_{L^{1}} \sim \lambda^{2 \delta_{j}},
$$

where $\sim$ denotes that equality holds only up to constants. Thus,

$$
\|\nabla u\|_{W^{\theta, 1}} \leq C\|\nabla u\|_{B V}^{\theta}\|\nabla u\|_{L^{1}}^{(1-\theta)} \leq C \lambda^{-\theta \sigma_{j}+(1-\theta) 2 \delta_{j}} .
$$

Requiring $\theta$ to be such that $-\theta \sigma_{j}+(1-\theta) 2 \delta_{j}=0$ then yields a bound on $\theta$. In our situations (i)-(iii) by rounding $\sigma_{j}, \delta_{j}$ up to the second digit after the comma, we obtain the lengths, area and regularity parameters shown in Figure 12. We remark that the aim of the works [RZZ16, RZZ17] was to establish some lower bounds on $\theta>0$ with an emphasis on the qualitative dependence on the choice of boundary datum $M$, but no focus on quantitative estimates. We further note that the cut-off at scales $10^{-4}, 10^{-9}$ amounts to a loss of very fine scale information. In particular, 


\begin{tabular}{l|lll} 
Case & $\delta$ & $\sigma$ & $\theta$ \\
\hline (i) center & 0.080 & 1.065 & 0.132 \\
(ii) corner & 0.035 & 1.032 & 0.062 \\
(iii) wall & 0.023 & 0.907 & 0.047 \\
\hline
\end{tabular}

Figure 12. This table shows the exponents of areas $\delta$ and length scales $\sigma$ and the resulting regularity exponent $\theta$ in the cases (i) (iii). The corresponding histograms in log-log format are given in Figures 13-15.
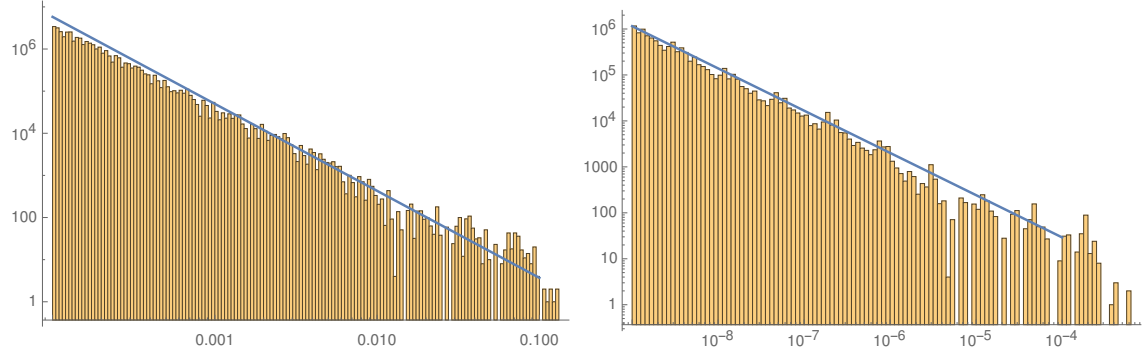

Figure 13. The distribution of lengths scales (left) and areas (right) for the boundary datum $M=0.33 e^{(1)}+0.33 e^{(2)}+0.34 e^{(3)}$ corresponding to case (i) are shown in log-log scale. The slopes of the interpolating lines, $-1-\sigma$ and $-1+\delta$, obtained by the least square method on the left $5 \%$ to $40 \%$ of the data, is given in Figure 12. The interpolation region here is chosen to avoid artifacts due to the cut-off and at large scales.
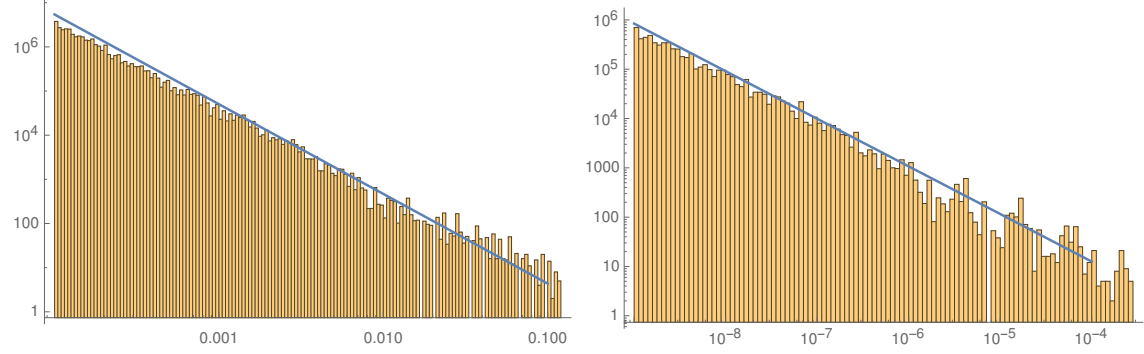

Figure 14. The distribution of lengths scales (left) and areas (right) for the boundary datum $M=0.2 e^{(1)}+0.2 e^{(2)}+0.6 e^{(3)}$ corresponding to case (ii) are shown in log-log scale.

we observe that with our length scale cut-off, the simulation in case (i) already covers roughly $50 \%$ of the area, while the one in the cases (ii) and (iii) only cover $15 \%$. Hence, the exponents derived in these cases might also have to be treated with caution. While the numerical method employed here in principle could also be applied to the construction of [RZZ17], as seen in Figure 11, there very small scales appear after already a small number of steps and no level sets reach the wells already after a finite number of steps. 

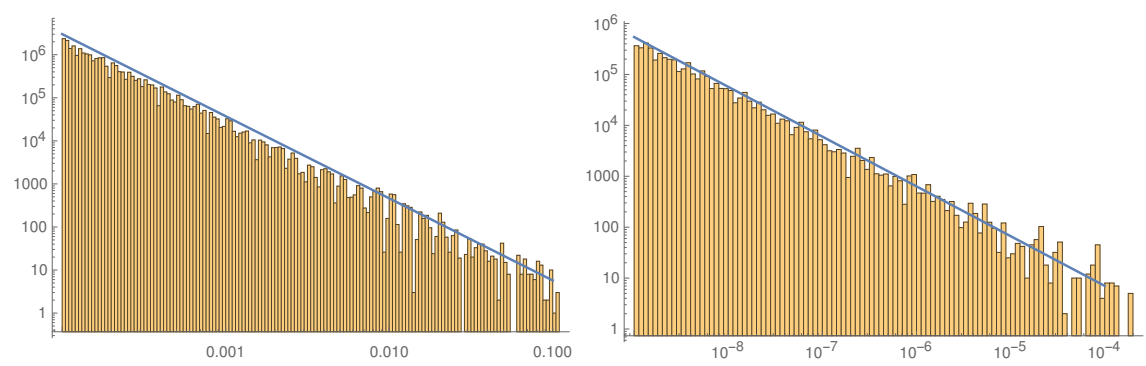

Figure 15. The distribution of lengths scales (left) and areas (right) for the boundary datum $M=0.449 e^{(1)}+0.451 e^{(2)}+0.1 e^{(3)}$ corresponding to case (iii) are shown in log-log scale.

\section{Appendix A. Fractional Poincaré Inequalities}

In this last section, for self-containedness, we recall a possible proof of the fractional Poincaré inequality (for fractional Besov type spaces), which is used in different forms in Section 2. We start with the $L^{2}$ based version:

Lemma A.1. Let $s \in(0,1)$ and $n \geq 1$. Let $\Omega \subset \mathbb{R}^{n}$ be an open, bounded $C^{1,1}$ domain. Let $u \in H^{s}\left(\mathbb{R}^{n}\right)$ with $\operatorname{supp}(u) \subset \bar{\Omega}$ and denote $\Omega_{\delta}=\{x \in \Omega: \operatorname{dist}(x, \partial \Omega) \geq$ $\delta\}$ for $\delta>0$. Then, there exists $C=C(s, \Omega, n)>1$ such that

$$
\|u\|_{L^{2}\left(\Omega_{\delta} \backslash \Omega_{2 \delta}\right)} \leq C \delta^{s}\|u\|_{H^{s}\left(\mathbb{R}^{n}\right)} .
$$

Proof. We use the Caffarelli-Silvestre extension $\bar{u}$ of $u$ [CS07], which is the $H^{1}\left(\mathbb{R}_{+}^{n+1}, x_{n+1}^{1-2 s}\right)$ solution to the equation

$$
\begin{aligned}
\nabla \cdot x_{n+1}^{1-2 s} \nabla \bar{u} & =0 \text { in } \mathbb{R}_{+}^{n+1}, \\
\bar{u} & =u \text { on } \mathbb{R}^{n} \times\{0\} .
\end{aligned}
$$

For $x_{1} \in \Omega_{\delta} \backslash \Omega_{2 \delta}, x_{2}=p\left(x_{1}\right) \in \mathbb{R}^{n} \backslash \bar{\Omega}$ with $\left|x_{1}-x_{2}\right| \leq 4 \delta$ and $\tilde{\delta} \in[\delta, 2 \delta]$, the fundamental theorem and Hölder's inequality yield

$$
\begin{aligned}
& \left|u\left(x_{1}\right)\right|^{2}=\left|\bar{u}\left(x_{1}, 0\right)\right|^{2} \leq 2\left|\bar{u}\left(x_{1}, \tilde{\delta}\right)\right|^{2}+2\left(\int_{0}^{\tilde{\delta}}\left|\partial_{t} \bar{u}\left(x_{1}, t\right)\right| d t\right)^{2} \\
& \leq 2\left|\bar{u}\left(x_{1}, \tilde{\delta}\right)\right|^{2}+C \tilde{\delta}^{2 s} \int_{0}^{\tilde{\delta}} t^{1-2 s}\left|\partial_{t} \bar{u}\left(x_{1}, t\right)\right|^{2} d t \\
& \leq 2\left|\bar{u}\left(p\left(x_{1}\right), \tilde{\delta}\right)\right|^{2}+C \tilde{\delta}^{2} \int_{0}^{1}\left|\nabla^{\prime} \bar{u}\left(\tau x_{1}+(1-\tau) p\left(x_{1}\right), \tilde{\delta}\right)\right|^{2} d \tau \\
& +C \tilde{\delta}^{2 s} \int_{0}^{\tilde{\delta}} t^{1-2 s}\left|\partial_{t} \bar{u}\left(x_{1}, t\right)\right|^{2} d t \\
& \leq 2 \tilde{\delta}^{2} \int_{0}^{1}\left|\nabla^{\prime} \bar{u}\left(\tau x_{1}+(1-\tau) p\left(x_{1}\right), \tilde{\delta}\right)\right|^{2} d \tau+C \tilde{\delta}^{2 s} \int_{0}^{\tilde{\delta}} t^{1-2 s}\left|\partial_{t} \bar{u}\left(x_{1}, t\right)\right|^{2} d t \\
& +C \tilde{\delta}^{2 s} \int_{0}^{\tilde{\delta}} t^{1-2 s}\left|\partial_{t} \bar{u}\left(p\left(x_{1}\right), t\right)\right|^{2} d t
\end{aligned}
$$


Here the constant $C>1$ changes from line to line and depends on $s$ but not on $\delta$. In deriving the above estimate, we applied the fundamental theorem thrice: First in the normal direction (where we then used Hölder's inequality to insert the weight $\left.t^{1-2 s}\right)$, then in the tangential directions and finally once more in the normal direction (where we used the vanishing Dirichlet data for $u\left(p\left(x_{1}\right)\right)=\bar{u}\left(p\left(x_{1}\right), 0\right)$ ). Estimating the integrals involving the normal derivative by using $\delta<\tilde{\delta} \leq 2 \delta$, we thus infer

$$
\begin{aligned}
\left|u\left(x_{1}\right)\right|^{2} \leq & 2 \tilde{\delta}^{2} \int_{0}^{1}\left|\nabla^{\prime} \bar{u}\left(\tau x_{1}+(1-\tau) p\left(x_{1}\right), \tilde{\delta}\right)\right|^{2} d \tau+C \delta^{2 s} \int_{0}^{2 \delta} t^{1-2 s}\left|\partial_{t} \bar{u}\left(x_{1}, t\right)\right|^{2} d t \\
& +C \delta^{2 s} \int_{0}^{2 \delta} t^{1-2 s}\left|\partial_{t} \bar{u}\left(p\left(x_{1}\right), t\right)\right|^{2} d t .
\end{aligned}
$$

Averaging over $\tilde{\delta} \in[\delta, 2 \delta]$ yields

$$
\begin{aligned}
\left|u\left(x_{1}\right)\right|^{2} \leq & 2 \delta^{-1} \int_{\delta}^{2 \delta} \tilde{\delta}^{2} \int_{0}^{1}\left|\nabla^{\prime} \bar{u}\left(\tau x_{1}+(1-\tau) p\left(x_{1}\right), \tilde{\delta}\right)\right|^{2} d \tau d \tilde{\delta}+C \delta^{2 s} \int_{0}^{2 \delta} t^{1-2 s}\left|\partial_{t} \bar{u}\left(x_{1}, t\right)\right|^{2} d t \\
& +C \delta^{2 s} \int_{0}^{2 \delta} t^{1-2 s}\left|\partial_{t} \bar{u}\left(p\left(x_{1}\right), t\right)\right|^{2} d t \\
= & 2 \delta^{-1} \int_{\delta}^{2 \delta} \tilde{\delta}^{1+2 s} \tilde{\delta}^{1-2 s} \int_{0}^{1}\left|\nabla^{\prime} \bar{u}\left(\tau x_{1}+(1-\tau) p\left(x_{1}\right), \tilde{\delta}\right)\right|^{2} d \tau d \tilde{\delta}+C \delta^{2 s} \int_{0}^{2 \delta} t^{1-2 s}\left|\partial_{t} \bar{u}\left(x_{1}, t\right)\right|^{2} d t \\
& +C \delta^{2 s} \int_{0}^{2 \delta} t^{1-2 s}\left|\partial_{t} \bar{u}\left(p\left(x_{1}\right), t\right)\right|^{2} d t \\
\leq & C \delta^{-1} \delta^{1+2 s} \int_{\delta}^{2 \delta} \tilde{\delta}^{1-2 s} \int_{0}^{1}\left|\nabla^{\prime} \bar{u}\left(\tau x_{1}+(1-\tau) p\left(x_{1}\right), \tilde{\delta}\right)\right|^{2} d \tau d \tilde{\delta}+C \delta^{2 s} \int_{0}^{2 \delta} t^{1-2 s}\left|\partial_{t} \bar{u}\left(x_{1}, t\right)\right|^{2} d t \\
& +C \delta^{2 s} \int_{0}^{2 \delta} t^{1-2 s}\left|\partial_{t} \bar{u}\left(p\left(x_{1}\right), t\right)\right|^{2} d t \\
\leq & C \delta^{2 s} \int_{0}^{2 \delta} \int_{0}^{1} t^{1-2 s}\left|\nabla^{\prime} \bar{u}\left(\tau x_{1}+(1-\tau) p\left(x_{1}\right), t\right)\right|^{2} d \tau d t+C \delta^{2 s} \int_{0}^{2 \delta} t^{1-2 s}\left|\partial_{t} \bar{u}\left(x_{1}, t\right)\right|^{2} d t \\
& +C \delta^{2 s} \int_{0}^{1-2 s}\left|\partial_{t} \bar{u}\left(p\left(x_{1}\right), t\right)\right|^{2} d t .
\end{aligned}
$$


Finally, integrating over $x_{1} \in \Omega_{\delta} \backslash \Omega_{2 \delta}$ leads to

$$
\begin{aligned}
\|u\|_{L^{2}\left(\Omega_{\delta} \backslash \Omega_{2 \delta}\right)}^{2} \leq & C \delta^{2 s} \int_{0}^{1} \int_{0}^{2 \delta} t^{1-2 s}\left\|\nabla^{\prime} \bar{u}(\tau \cdot+(1-\tau) p(\cdot), t)\right\|_{L^{2}\left(\Omega_{\delta} \backslash \Omega_{2 \delta}\right)}^{2} d t d \tau \\
& +2 C \delta^{2 s}\left\|t^{\frac{1-2 s}{2}} \partial_{t} \bar{u}\right\|_{L^{2}\left(\left(\Omega_{\delta} \backslash \Omega_{2 \delta}\right) \times[0,2 \delta]\right)}^{2} \\
\leq & C \delta^{2 s}\left\|t^{\frac{1-2 s}{2}} \nabla \bar{u}\right\|_{L^{2}(\Omega \times[0,2 \delta])}^{2} \\
\leq & C \delta^{2 s}\left\|t^{\frac{1-2 s}{2}} \nabla \bar{u}\right\|_{L^{2}\left(\mathbb{R}_{+}^{n+1}\right)}^{2} .
\end{aligned}
$$

Hence, using that (see [CS07])

$$
\left\|t^{\frac{1-2 s}{2}} \nabla \bar{u}\right\|_{L^{2}\left(\mathbb{R}_{+}^{n+1}\right)}^{2} \leq C\|u\|_{\dot{H}^{s}\left(\mathbb{R}^{n}\right)}
$$

we obtain the desired estimate (70).

Similarly, we have the analogue of this in Sobolev and Besov spaces:

Lemma A.2. Let $s \in(0,1), p \in(1, \infty)$ and $n \geq 1$. Let $\Omega \subset \mathbb{R}^{n}$ be an open, bounded $C^{1,1}$ domain. Let $u \in W^{s, p}\left(\mathbb{R}^{n}\right)$ with $\operatorname{supp}(u) \subset \bar{\Omega}$ and denote $\Omega_{\delta}=\{x \in$ $\Omega: \operatorname{dist}(x, \partial \Omega) \geq \delta\}$ for $\delta>0$. Then, there exists $C=C(s, p, \Omega, n)>1$ such that

$$
\|u\|_{L^{p}\left(\Omega_{\delta} \backslash \Omega_{2 \delta}\right)} \leq C \delta^{s}\|u\|_{W^{s, p}\left(\mathbb{R}^{n}\right)} .
$$

Proof. The argument follows essentially as above. However for the estimate in the normal direction, we use

$$
\begin{aligned}
\left|\bar{u}\left(x_{1}, 0\right)\right|^{p} & \leq C\left|\bar{u}\left(x_{1}, \delta\right)\right|^{p}+C\left(\int_{0}^{\delta} \partial_{t} \bar{u} d t\right)^{p} \\
& \leq C\left|\bar{u}\left(x_{1}, \delta\right)\right|^{p}+C \int_{0}^{\delta}\left|t^{1-\frac{1}{p}-s} \partial_{t} \bar{u}\right|^{p} d t\left(\int_{0}^{\delta} t^{-1+\frac{s p}{p-1}} d t\right)^{p-1} \\
& \leq C\left|\bar{u}\left(x_{1}, \delta\right)\right|^{p}+C \delta^{s p} \int_{0}^{\delta} t^{p-1-s p}\left|\partial_{t} \bar{u}\right|^{p} d t
\end{aligned}
$$

Combining this with estimates in the tangential directions similarly as in the proof of Lemma A.2, we obtain

$$
\|u\|_{L^{p}\left(\Omega_{\delta} \backslash \Omega_{2 \delta}\right)} \leq C \delta^{s}\left\|t^{1-\frac{1}{p}-s} \nabla \bar{u}\right\|_{L^{p}\left(\mathbb{R}_{+}^{n+1}\right)} \leq C \delta^{s}\|u\|_{W^{s, p}\left(\mathbb{R}^{n}\right)} .
$$

Here we used the trace characterisation of $W^{s, p}\left(\mathbb{R}^{n}\right)$, see [LS16, Section 10], where the authors rely on the characterisation from [BC15].

A similar argument using the characterisation of Besov spaces from [LS16, Section 10] (where the authors again rely on the characterisation from [BC15]) also yields a similar Poincaré estimate in Besov spaces.

\section{Appendix B. Pseudocode Convex Integration Algorithm}

In order to allow for a self-contained and more precise understanding of the algorithms used in generating the pictures from above, we include a pseudocode variant of the code which we have used. In the sequel, we make use of the convention that the $i$-th component of a function $f$ is described by $f[i]$.

Algorithm B.1. Let $M \in \mathcal{M}:=\left\{N \in \mathbb{R}^{2 \times 2}: e(N) \in \operatorname{intconv}\left(\left\{e^{(1)}, e^{(2)}, e^{(3)}\right\}\right)\right\}$, where $e^{(1)}, e^{(2)}, e^{(3)}$ are as in (68). Let $\Omega \subset \mathbb{R}^{2}$ be a triangle. 
(1a) Variables. We consider

- the displacement $u_{k}: \Omega \rightarrow \mathbb{R}^{2}$ at step $k$,

- a collection of (up to null-sets disjoint) triangles $\widehat{\Omega}_{k}=\left\{\Omega_{1}^{k}, \ldots, \Omega_{j_{k}}^{k}\right\}$, which cover $\Omega$,

- and the error in matrix space $\epsilon_{k}: \widehat{\Omega}_{k} \rightarrow(0,1)$ at step $k$, which is constant on each subset of $\widehat{\Omega}_{k}$.

(1b) Functions. We consider a "covering function", which covers a given triangle by "good sets", on which the deformation $u_{k}$ will be improved, and a "remainder". More precisely,

$\operatorname{Cover}_{v}: \mathcal{T} \times \mathcal{M} \rightarrow \mathcal{R} \times \mathcal{T}$

$$
(T, M) \mapsto\left(\left\{Q_{1}, \ldots, Q_{j(T, M)}\right\},\left\{T_{1}, \ldots, T_{l(T, M)}\right\}\right) .
$$

Here $\mathcal{T}$ is the set of all triangles, $\mathcal{R}$ is a set of certain quadrilaterals (these differ in [RZZ16] and [RZZ17]) and $\mathcal{M}:=\left\{M \in \mathbb{R}^{2 \times 2}: e(M) \in\right.$ $\left.\operatorname{intconv}\left(\left\{e^{(1)}, e^{(2)}, e^{(3)}\right\}\right)\right\}$. For each $T \in \mathcal{T}$ and $M \in \mathcal{M}$ we have an (up to null sets) disjoint covering

$$
T=\bigcup_{Q \in \operatorname{Cover}_{v}(T, M)[1]} Q \cup \underset{\tilde{T} \in \operatorname{Cover}_{v}(T, M)[2]}{\bigcup} \tilde{T},
$$

with

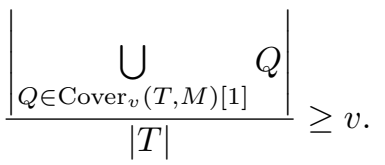

The sets $Q \in \operatorname{Cover}_{v}(T, M)[1]$ are the "good sets", on which the current displacement gradient $\nabla u_{k}$ will be modified and pushed towards the energy wells. The sets $\tilde{T} \in \operatorname{Cover}_{v}(T, M)[2]$ are the "remainders" on which the displacement $u_{k}$ is not changed.

We further consider a "replacement function", which improves the current displacement gradient in the sense that the replaced deformation gradient is closer to the wells (or at least closer to the wells on a large portion of the domain). This depends on the current displacement gradient, the underlying domain and the error in matrix space. As an output it yields

- the level sets (in the form of a finite collection of triangles) of the new improved deformation,

- a piecewise affine function whose symmetric gradient attains values in $\operatorname{int} \operatorname{conv}\left(\left\{e^{(1)}, e^{(2)}, e^{(3)}\right\}\right)$,

- and an updated error in matrix space.

More precisely,

$$
\begin{aligned}
& \text { Replace }: \mathcal{R} \times \mathcal{A}_{\operatorname{intconv}\left(\left\{e^{(1)}, e^{(2)}, e^{(3)}\right\}\right)} \times(0,1) \\
& \rightarrow \mathcal{T} \times \mathcal{A}_{\operatorname{intconv}\left(\left\{e^{(1)}, e^{(2)}, e^{(3)}\right\}\right)} \times(0,1), \\
&\left(Q, w_{\text {old }}, \epsilon\right) \mapsto\left(\left\{T_{1}, \ldots, T_{j_{0}}\right\}, w, \tilde{\epsilon}\right)
\end{aligned}
$$

Here $\mathcal{A}_{\text {intconv }\left(\left\{e^{(1)}, e^{(2)}, e^{(3)}\right\}\right)}$ denotes the set of piecewise affine deformations with symmetric gradients in intconv $\left\{e^{(1)}, e^{(2)}, e^{(3)}\right\}$. Furthermore, $\left.w\right|_{\partial Q}=$ $\left.w_{\text {old }}\right|_{\partial Q}$.

(2) Initialization. We begin by setting $u_{0}(x)=M x, \epsilon_{0}=\epsilon_{0}(M), \widehat{\Omega}_{0}=\{\Omega\}$.

(3) Iteration step. The algorithm proceeds iteratively: Assume that $u_{k}, \epsilon_{k}$ and $\widehat{\Omega}_{k}$ with $k \geq 0$ are already given. Then on each $\Omega_{j}^{k} \in \widehat{\Omega}_{k}$ for which $\left.e\left(\nabla u_{k}\right)\right|_{\Omega_{j}^{k}} \notin\left\{e^{(1)}, e^{(2)}, e^{(3)}\right\}$, apply the function $\operatorname{Cover}_{v}\left(\Omega_{j}^{k},\left.\nabla u_{k}\right|_{\Omega_{j}^{k}}\right)$. Let $\operatorname{Cover}_{v}\left(\Omega_{j}^{k},\left.\nabla u_{k}\right|_{\Omega_{j}^{k}}\right)[1]=\left\{\Omega_{j, 1}^{k}, \ldots, \Omega_{j, m(j, k)}^{k}\right\}$. 
For each $\Omega_{j, m}^{k} \in \operatorname{Cover}_{v}\left(\Omega_{j}^{k},\left.\nabla u_{k}\right|_{\Omega_{j}^{k}}\right)[1]$ apply the function $\operatorname{Replace}\left(\Omega_{j, m}^{k},\left.u_{k}\right|_{\Omega_{j}^{k}}, \epsilon_{k}\right)$. This yields

(i) an up to null-sets disjoint covering of $\Omega_{j, m}^{k}$ into triangles

$$
\left\{\Omega_{j, m, 1}^{k}, \ldots, \Omega_{j, m, l(j, m, k)}^{k}\right\}:=\operatorname{Replace}\left(\Omega_{j, m}^{k},\left.u_{k}\right|_{\Omega_{j}^{k}}, \epsilon_{k}\right)[1] ;
$$

(ii) a function $v_{j, m, k}:=\operatorname{Replace}\left(\Omega_{j, m}^{k},\left.u_{k}\right|_{\Omega_{j}^{k}}, \epsilon_{k}\right)[2]: \Omega_{j, m}^{k} \rightarrow \mathbb{R}^{2}$ whose gradient is constant on each of the sets $\Omega_{j, m, l}^{k}$ with $l \in\{1, \ldots, l(j, m, k)\}$ and for which $v_{j, m, k}(x)=u_{k}(x)$ for all $x \in \partial \Omega_{j, m}^{k}$;

(iii) a parameter $\tilde{\epsilon}_{j, m, k}:=\operatorname{Replace}\left(\Omega_{j, m}^{k},\left.u_{k}\right|_{\Omega_{j}^{k}}, \epsilon_{k}\right)[3]$.

We then set

$$
\begin{aligned}
& \left.u_{k+1}\right|_{\Omega_{j, m}^{k}}=v_{j, m, k} \text { and }\left.u_{k+1}\right|_{\operatorname{Cover}_{v}\left(\Omega_{j}^{k},\left.\nabla u_{k}\right|_{\Omega_{j}^{k}}\right)[2]}=\left.u_{k}\right|_{\operatorname{Cover}_{v}\left(\Omega_{j}^{k},\left.\nabla u_{k}\right|_{\Omega_{j}^{k}}\right)[2]}, \\
& \widetilde{\Omega}_{k+1}=\bigcup_{j, m} \operatorname{Replace}\left(\Omega_{j, m}^{k},\left.u\right|_{\Omega_{j}^{k}}, \epsilon_{k}\right)[1] \cup \bigcup_{j} \operatorname{Cover}_{v}\left(\Omega_{j}^{k},\left.\nabla u_{k}\right|_{\left.\Omega_{j}^{k}\right)}[2],\right. \\
& \left.\epsilon_{k+1}\right|_{T}= \begin{cases}\tilde{\epsilon}_{j, m, k} & \text { if } T \subset \Omega_{j, m, l}^{k} \in \operatorname{Cover}_{v}\left(\Omega_{j}^{k},\left.\nabla u_{k}\right|_{\Omega_{j}^{k}}\right)[1] \text { for some } j \in\left\{1, \ldots, j_{k}\right\}, \\
\left.\epsilon_{k}\right|_{T} & \text { if } T \in \operatorname{Cover}_{v}\left(\Omega_{j}^{k},\left.\nabla u_{k}\right|_{\Omega_{j}^{k}}\right)[2] \text { for some } j \in\left\{1, \ldots, j_{k}\right\} .\end{cases}
\end{aligned}
$$

If on $\Omega_{j}^{k}$ we already have $\left.e\left(\nabla u_{k}\right)\right|_{\Omega_{j}^{k}} \in\left\{e^{(1)}, e^{(2)}, e^{(3)}\right\}$, we set $\left.u_{k+1}\right|_{\Omega_{j}^{k}}=$ $\left.u_{k}\right|_{\Omega_{j}^{k}}$ and

$$
\widehat{\Omega}_{k+1}=\widetilde{\Omega}_{k+1} \cup\left\{\Omega_{j}^{k} \in \widehat{\Omega}_{k}:\left.\nabla u_{k}\right|_{\Omega_{j}^{k}} \in\left\{e^{(1)}, e^{(2)}, e^{(3)}\right\} \text { a.e. }\right\} .
$$

It has been shown in [RZZ16] and [RZZ17] that this procedure is well-defined, if the function Replace is chosen appropriately (there is a slight subtlety in that in those articles we approximate domains by rectangles, but this does not matter for the non-quantitative algorithm which is used here). As explained above, in spite of their similar overall structure, the algorithms from [RZZ16, RZZ17] however differ substantially in their underlying replacement constructions encoded by the function Replace. The function $\operatorname{Cover}_{v}$ is essentially given by a greedy algorithm.

\section{REFERENCES}

[ABK15] Virginia Agostiniani, Timothy Blass, and Konstantinos Koumatos. From nonlinear to linearized elasticity via $\Gamma$-convergence: The case of multiwell energies satisfying weak coercivity conditions. Mathematical Models and Methods in Applied Sciences, 25(01):1-38, 2015.

[Bal76] John M Ball. Convexity conditions and existence theorems in nonlinear elasticity. Archive for rational mechanics and Analysis, 63(4):337-403, 1976.

[Bal02] John M Ball. Some open problems in elasticity. In Geometry, mechanics, and dynamics, pages 3-59. Springer, 2002.

[Bal04] John M. Ball. Mathematical models of martensitic microstructure. Materials Science and Engineering: A, 378(1-2):61 - 69, 2004. European Symposium on Martensitic Transformation and Shape-Memory.

[BC15] Huy-Qui Bui and Timothy Candy. A characterisation of the Besov-Lipschitz and Triebel-Lizorkin spaces using Poisson like kernels. arXiv preprint arXiv:1502.06836, 2015.

[BCD11] Hajer Bahouri, Jean-Yves Chemin, and Raphaël Danchin. Fourier analysis and nonlinear partial differential equations, volume 343. Springer Science \& Business Media, 2011.

[BCH15] John M Ball, Pierluigi Cesana, and Ben Hambly. A probabilistic model for martensitic avalanches. In MATEC Web of Conferences, volume 33. EDP Sciences, 2015.

[BDLSJV17] Tristan Buckmaster, Camillo De Lellis, László Székelyhidi Jr, and Vlad Vicol. Onsager's conjecture for admissible weak solutions. arXiv preprint arXiv:1701.08678, 2017. 
[Bha93] Kaushik Bhattacharya. Comparison of the geometrically nonlinear and linear theories of martensitic transformation. Continuum Mechanics and Thermodynamics, 5(3):205-242, 1993.

[Bha03] Kaushik Bhattacharya. Microstructure of martensite: why it forms and how it gives rise to the shape-memory effect Oxford series on materials modeling. Oxford University Press, 2003.

[BJ89] John M. Ball and Richard D. James. Fine phase mixtures as minimizers of energy. In Analysis and Continuum Mechanics, pages 647-686. Springer, 1989.

[BM01] Haïm Brezis and Petru Mironescu. Gagliardo-Nirenberg, composition and products in fractional Sobolev spaces. Journal of Evolution Equations, 1(4):387-404, 2001.

[BMC09] John M Ball and Carlos Mora-Corral. A variational model allowing both smooth and sharp phase boundaries in solids. Commun. Pure Appl. Anal, 8(1):55-81, 2009.

[CC15] Allan Chan and Sergio Conti. Energy scaling and branched microstructures in a model for shape-memory alloys with so (2) invariance. Mathematical Models and Methods in Applied Sciences, 25(06):1091-1124, 2015.

[CDLS12] Sergio Conti, Camillo De Lellis, and László Székelyhidi. h-principle and rigidity for $C^{1, \alpha}$ isometric embeddings. In Nonlinear partial differential equations, pages 83116. Springer, 2012.

[CH18] Pierluigi Cesana and Ben Hambly. A probabilistic model for interfaces in a martensitic phase transition. arXiv preprint arXiv:1810.04380, 2018.

[CKZ17] S Conti, M Klar, and B Zwicknagl. Piecewise affine stress-free martensitic inclusions in planar nonlinear elasticity. Proc. R. Soc. A, 473(2203):20170235, 2017.

[CO09] Antonio Capella and Felix Otto. A rigidity result for a perturbation of the geometrically linear three-well problem. Communications on Pure and Applied Mathematics, 62(12):1632-1669, 2009.

[CO12] Antonio Capella and Felix Otto. A quantitative rigidity result for the cubic-totetragonal phase transition in the geometrically linear theory with interfacial energy. Proceedings of the Royal Society of Edinburgh: Section A Mathematics, 142, pp 273-327 doi:10.1017/S0308210510000478, 2012.

[Con00] Sergio Conti. Branched microstructures: scaling and asymptotic self-similarity. Comm. Pure Appl. Math, 53(11):1448-1474, 2000.

[CPL14] Pierluigi Cesana, Marcel Porta, and Turab Lookman. Asymptotic analysis of hierarchical martensitic microstructure. Journal of the Mechanics and Physics of Solids, 72:174-192, 2014.

[CS07] Luis Caffarelli and Luis Silvestre. An extension problem related to the fractional Laplacian. Communications in partial differential equations, 32(8):1245-1260, 2007.

[DKMŠ00] Georg Dolzmann, Bernd Kirchheim, Stefan Müller, and Vladimír Šverák. The twowell problem in three dimensions. Calculus of Variations and Partial Differential Equations, 10:21-40, 2000. 10.1007/PL00013455.

[DM95a] Georg Dolzmann and Stefan Müller. The influence of surface energy on stressfree microstructures in shape memory alloys. Meccanica, 30:527-539, 1995. $10.1007 /$ BF01557083.

[DM95b] Georg Dolzmann and Stefan Müller. Microstructures with finite surface energy: the two-well problem. Archive for Rational Mechanics and Analysis, 132:101-141, 1995.

[DM12] Bernard Dacorogna and Paolo Marcellini. Implicit partial differential equations, volume 37. Springer Science \& Business Media, 2012.

[DMNP02] Gianni Dal Maso, Matteo Negri, and Danilo Percivale. Linearized elasticity as $\gamma$-limit of finite elasticity. Set-Valued Analysis, 10(2-3):165-183, 2002.

[DMP08a] Bernard Dacorogna, Paolo Marcellini, and Emanuele Paolini. An explicit solution to a system of implicit differential equations. In Annales de l'Institut Henri Poincare (C) Non Linear Analysis, volume 25, pages 163-171. Elsevier, 2008.

[DMP08b] Bernard Dacorogna, Paolo Marcellini, and Emanuele Paolini. Lipschitz-continuous local isometric immersions: rigid maps and origami. Journal de mathématiques pures et appliquées, 90(1):66-81, 2008.

[DMP10] Bernard Dacorogna, Paolo Marcellini, and Emanuele Paolini. Origami and partial differential equations. Notices of $A M S, 57(5): 598-606,2010$.

[Fri95] Uriel Frisch. Turbulence: the legacy of AN Kolmogorov. Cambridge university press, 1995.

[Gro73] Mikhail Leonidovich Gromov. Convex integration of differential relations. i. Izvestiya: Mathematics, 7(2):329-343, 1973.

[Ina] Tomonari Inamura. Martensitic material. https://www.newton.ac.uk/event/dnm. An experiment from experiment of Tomonari Inamura's group, Accessed: 201801-08. 
[Ise16] Philip Isett. A proof of Onsager's conjecture. arXiv preprint arXiv:1608.08301, 2016.

[Kha13] Armen G Khachaturyan. Theory of structural transformations in solids. Courier Corporation, 2013.

[Kir98] Bernd Kirchheim. Lipschitz minimizers of the 3-well problem having gradients of bounded variation. MPI preprint, 1998.

[Kir03] Bernd Kirchheim. Rigidity and geometry of microstructures. MPI-MIS lecture notes, 2003.

[KK91] Y. Kitano and K. Kifune. HREM study of disclinations in MgCd ordered alloy. Ultramicroscopy, 39(1-4):279-286, 1991.

[KKO13] Hans Knüpfer, Robert V Kohn, and Felix Otto. Nucleation barriers for the cubic-totetragonal phase transformation. Communications on pure and applied mathematics, 66(6):867-904, 2013.

[KM94] Robert V Kohn and Stefan Müller. Surface energy and microstructure in coherent phase transitions. Communications on Pure and Applied Mathematics, 47(4):405435, 1994.

[LS16] Enno Lenzmann and Armin Schikorra. Sharp commutator estimates via harmonic extensions. arXiv preprint arXiv:1609.08547, 2016.

[MŠ98] Stefan Müller and Vladimir Šverák. Unexpected solutions of first and second order partial differential equations. In International Congress, page 691, 1998.

[MŠ99] Stefan Müller and Vladimír Šverák. Convex integration with constraints and applications to phase transitions and partial differential equations. Journal of the European Mathematical Society, 1:393-422, 1999. 10.1007/s100970050012.

[Mü199] Stefan Müller. Variational models for microstructure and phase transitions. In Calculus of variations and geometric evolution problems, pages 85-210. Springer, 1999.

[OK90] Bohumír Opic and Alois Kufner. Hardy-type inequalities, volume 219. Halsted Press, 1990.

[Ped97] Pablo Pedregal. Parametrized measures and variational principles, volume 30. Birkhauser Basel, 1997

[PLKK97] A Yu Pasko, AA Likhachev, Yu N Koval, and VI Kolomytsev. 2d Fourier analysis and its application to study of scaling properties and fractal dimensions of $\varepsilon$-martensite distribution in $\gamma$-matrix of Fe-Mn-Si alloy. Le Journal de Physique IV, 7(C5):C5435, 1997

[Rül16a] Angkana Rüland. The cubic-to-orthorhombic phase transition: Rigidity and nonrigidity properties in the linear theory of elasticity. Archive for Rational Mechanics and Analysis, 221(1):23-106, 2016.

[Rül16b] Angkana Rüland. A rigidity result for a reduced model of a cubic-to-orthorhombic phase transition in the geometrically linear theory of elasticity. Journal of Elasticity, 123(2):137-177, 2016.

[RZZ16] Angkana Rüland, Christian Zillinger, and Barbara Zwicknagl. Higher Sobolev regularity of convex integration solutions in elasticity. arXiv preprint arXiv:1610.02529, 2016.

[RZZ17] Angkana Rüland, Christian Zillinger, and Barbara Zwicknagl. Higher Sobolev regularity of convex integration solutions in elasticity: The Dirichlet problem with affine data in $\operatorname{int}\left(k^{l c}\right)$. arXiv preprint arXiv:1709.02880, 2017.

[Sic99] Winfried Sickel. Pointwise multipliers of Lizorkin-Triebel spaces. In The Maz'ya anniversary collection, pages 295-321. Springer, 1999.

[Sim17] Thilo Simon. Rigidity of branching microstructures in shape memory alloys. arXiv preprint arXiv:1705.03664, 2017.

[SJ12] László Székelyhidi Jr. From isometric embeddings to turbulence. MPI lecture notes, 2012.

[Tao06] Terence Tao. Nonlinear dispersive equations: local and global analysis. Number 106. American Mathematical Soc., 2006.

[TIVP17] Genís Torrents, Xavier Illa, Eduard Vives, and Antoni Planes. Geometrical model for martensitic phase transitions: Understanding criticality and weak universality during microstructure growth. Physical Review E, 95(1):013001, 2017.

[Tri06] Hans Triebel. Theory of function spaces. III, volume 100 of Monographs in Mathematics. BirkhauserVerlag, Basel, 2006.

MaX-Planck-Institute for Mathematics in the Sciences, Inselstrasse 22, 04103 Leipzig, GERMANY

E-mail address: rueland@mis.mpg.de 
Department of Mathematics, University of Southern California, 3620 S. Vermont Avenue, Los Angeles, CA 90089-2532, US

E-mail address: zillinge@usc.edu

Basque Center for Applied Mathematics, Mazarredo, 14. 48009 Bilbao Basque CounTRY - SPAIN

E-mail address: jtaylor@bcamath.org 